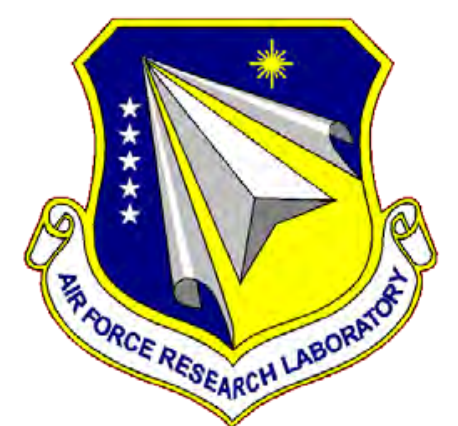

\title{
AFRL-RY-WP-TR-2015-0163
}

\section{THERMAL DIODES BASED ON NEAR-FIELD RADIATION}

Michal Lipson

Cornell University

OCTOBER 2015

Final Report

Approved for public release; distribution unlimited.

See additional restrictions described on inside pages

STINFO COPY

AIR FORCE RESEARCH LABORATORY SENSORS DIRECTORATE

WRIGHT-PATTERSON AIR FORCE BASE, OH 45433-7320

AIR FORCE MATERIEL COMMAND

UNITED STATES AIR FORCE 


\section{NOTICE AND SIGNATURE PAGE}

Using Government drawings, specifications, or other data included in this document for any purpose other than Government procurement does not in any way obligate the U.S. Government. The fact that the Government formulated or supplied the drawings, specifications, or other data does not license the holder or any other person or corporation; or convey any rights or permission to manufacture, use, or sell any patented invention that may relate to them.

This report is the result of contracted fundamental research deemed exempt from public affairs security and policy review in accordance with SAF/AQR memorandum dated 10 Dec 08 and AFRL/CA policy clarification memorandum dated 16 Jan 09. This report is available to the general public, including foreign nationals.

\section{AFRL-RY-WP-TR-2015-0163 HAS BEEN REVIEWED AND IS APPROVED FOR PUBLICATION IN ACCORDANCE WITH ASSIGNED DISTRIBUTION STATEMENT.}

// Signature//

MARK J. PACER, Program Manager

Devices for Sensing Branch

Aerospace Components \& Subsystems Division
// Signature//

ROSS W. DETTMER, Chief

Devices for Sensing Branch

Aerospace Components \& Subsystems Division

// Signature//

JASON V. PAUL, MAJOR, USAF

Deputy Division Chief

Aerospace Components \& Subsystems Division

Sensors Directorate

This report is published in the interest of scientific and technical information exchange, and its publication does not constitute the Government's approval or disapproval of its ideas or findings.

*Disseminated copies will show “//Signature//" stamped or typed above the signature blocks. 


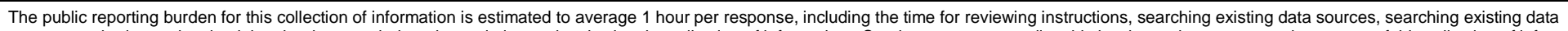

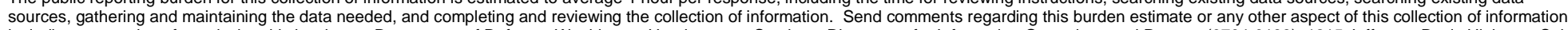

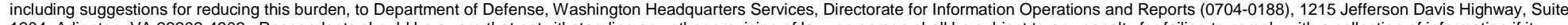

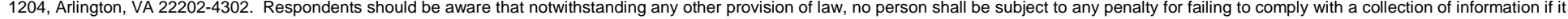
does not display a currently valid OMB control number. PLEASE DO NOT RETURN YOUR FORM TO THE ABOVE ADDRESS

\begin{tabular}{|c|c|}
\hline $\begin{array}{c}\text { 1. REPORT DATE (DD-MM-YY) } \\
\text { October } 2015\end{array}$ & $\begin{array}{c}\text { 2. REPORT TYPE } \\
\text { Final }\end{array}$ \\
\hline
\end{tabular}

4. TITLE AND SUBTITLE

THERMAL DIODES BASED ON NEAR-FIELD RADIATION
3. DATES COVERED (From - To)

31 January $2014-31$ July 2015

\section{AUTHOR(S)}

Michal Lipson

7. PERFORMING ORGANIZATION NAME(S) AND ADDRESS(ES)

Cornell University

373 Pine Tree Road

Ithaca, NY 14850-2820

9. SPONSORING/MONITORING AGENCY NAME(S) AND ADDRESS(ES)

Air Force Research Laboratory

Sensors Directorate

Wright-Patterson Air Force Base, OH 45433-7320

Air Force Materiel Command

United States Air Force 5a. CONTRACT NUMBER

FA8650-14-1-7406

5b. GRANT NUMBER

5c. PROGRAM ELEMENT NUMBER

61101E

5d. PROJECT NUMBER

1000

5e. TASK NUMBER

$\mathrm{N} / \mathrm{A}$

5f. WORK UNIT NUMBER

Y0ZF

8. PERFORMING ORGANIZATION REPORT NUMBER

AFRL-RY-WP-TR-2015-0163

10. SPONSORING/MONITORING AGENCY ACRONYM(S)

AFRL/RYDD

Research Projects Agency 675 North Randolph Street Arlington, VA 22203-2114
11. SPONSORING/MONITORING AGENCY REPORT NUMBER(S) AFRL-RY-WP-TR-2015-0163

12. DISTRIBUTION/AVAILABILITY STATEMENT

Approved for public release; distribution unlimited

13. SUPPLEMENTARY NOTES

This material is based on research sponsored by Air Force Research Laboratory (AFRL) and the Defense Advanced

Research Agency (DARPA) under agreement number FA8650-14-1-7406. The U.S. Government is authorized to reproduce and distribute reprints for Governmental purposes notwithstanding any copyright notation thereon. The views and conclusions contained herein are those of the authors and should not be interpreted as necessarily representing the official policies or endorsements, either expressed or implied, of Air Force Research Laboratory (AFRL) and the Defense Advanced Research Agency (DARPA) or the U.S. Government. This report is the result of contracted fundamental research deemed exempt from public affairs security and policy review in accordance with SAF/AQR memorandum dated 10 Dec 08 and AFRL/CA policy clarification memorandum dated 16 Jan 09. Report contains color.

14. ABSTRACT

The objectives of this work were to demonstrate a thermal rectification phenomenon: non-reciprocal heat transfer between two structures. This effect could be used to maintain the temperature of a device at a lower temperature than its surrounding, unaffected from neighboring hotspots. We proposed to demonstrate experimentally thermal rectification of $>40 \%$ based on our recent demonstration of near-field heat transfer. Simulation with alumina, silica and platinum coated nano-beams showing $\sim 65 \%$ rectification was achieved. The demonstration of thermal rectification $>40 \%$ between the two nano-membranes was not achieved but potential avenues identified. The current platform, with slight modifications in the deposited layers on the nano-beam surface, is suitable to obtain required separation for near field heat transfer and eventually thermal rectification of $>40 \%$.

15. SUBJECT TERMS

nano-membranes, thermal rectification, diodes, near-field radiation

16. SECURITY CLASSIFICATION OF:

a. REPORT

b. ABSTRACT

c. THIS PAGE

Unclassified
17. LIMITATION OF ABSTRACT: SAR
18. NUMBER OF PAGES 48 19a. NAME OF RESPONSIBLE PERSON (Monitor) Mark Pacer

19b. TELEPHONE NUMBER (Include Area Code) $\mathrm{N} / \mathrm{A}$ 


\section{TABLE OF CONTENTS}

$\begin{array}{lll}\text { Section } & \text { Page }\end{array}$

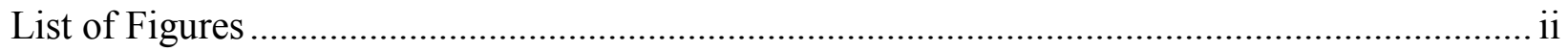

1.0 Summary and Importance of Main Achievements ........................................................... 1

2.0 Technical Details of Main Achievement …………………………………………..... 3

3.0 Milestones Achieved During the Project …………………………………………..... 15

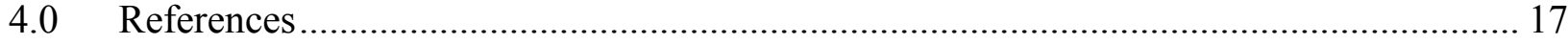

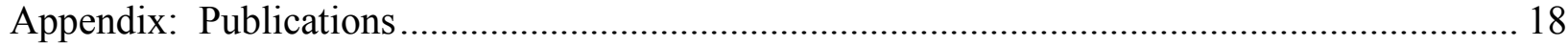

List of Acronyms, Abbreviations, and Symbols ....................................................................... 43 


\section{List of Figures}

Figure

Page

Figure 1: Schematic and Theoretical Prediction

Figure 2: (a) SEMs of the Device and (b) False Color SEM of the Nano-beams Cross

Section Prior to Substrate Removal

Figure 3: (a) Measured Temperature and (b) Heat Transfer Power............................................ 5

Figure 4: Schematic Representation of the Operation Principle of a Thermal Rectifier .............. 6

Figure 5: (a) Measurement of the Phonon Resonances of SiC and Comparison with

Theory and (b) Predicted Surface Energy Density of our Deposited SiC Film, Compared to that of $\mathrm{SiO}_{2}$, which was the Material used in our Previous Platform .................................... 7

Figure 6: Improved Mechanical Design for Near-field Rectification Measurement .................... 8

Figure 7: SEM Showing Detrimental Bending of the Device when Fabricated in $130 \mathrm{~nm}$

Thick Silicon Carbide .................................................................................................... 9

Figure 8: SEM of the Fabricated Device Based on a $\mathrm{Si}_{3} \mathrm{~N}_{4}$ Mechanical Support Coated

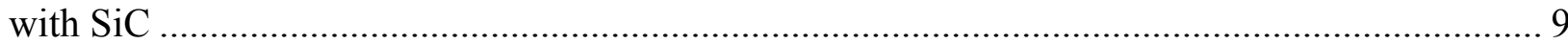

Figure 9: Measured MEMS Displacement as a Function of the Voltage on the MEMS Actuators 10

Figure 10: (a) Measurement of Near-field Heat Transfer as a Function of the Distance Between the Nano-beams (the far-field limit is included in the inset (log scale)) and

(b) Minimum Achieved Gap (red) and Enhancement over Far-field (blue) as a Function of the Heated Beam Temperature

Figure 11: Normalized Rectification as a Function of Film Thickness for Measured Damping Coefficient of our Polycrystalline $\operatorname{SiC}\left(\Gamma=9.5 \mathrm{~cm}^{-1}\right)$. 12

Figure 12: Heat Transfer between Thin Pt and $\mathrm{SiO}_{2}$ as a Function of Pt Film Thickness for Two Different Temperatures and Normalized by Black Body ....

Figure 13: (a) Multilayer Stack of Materials on Two Nano-beam Surfaces for Efficient Heat Transfer and (b) Angled Evaporation of Currently Fabricated Nano-beams to allow the Required Multilayer Stack on the Inner Heat Exchanging Surfaces 13

Figure 14: Heat Transfer Spectrum for the Structure Shown in Figure 12a with 40nm Gap..... 


\subsection{Summary and Importance of Main Achievements}

In this final report we summarize the work done towards demonstration of rectification, various milestones achieved during the process, and the current status of the work.

During the first part of this project we designed and demonstrated a platform for measurement of near-field radiative heat transfer on-chip. A strong on-chip radiative heat transfer was shown using a novel geometrical configuration of two parallel suspended nano-beams with silicon nitride core. The distance between the two nano-beams was tuned electrostatically and the heat transfer was measured using an integrated platinum resistor. We demonstrated $107 \mathrm{nW} / \mathrm{K}$ of background heat conduction on the nano-beams, thermally isolated from environment with $<20 \mathrm{~nm}$ precision in position control. (Milestones achieved: I.1, I.2, I.3, and I.4)

Later, in the second part, we performed simulations and found that silicon carbide ( $\mathrm{SiC}$ ) nano-beam could allow near-field rectification of heat transfer. We proposed to coast one of the nano-beam in our design with thin layer of silicon oxide. A thin layer of silicon oxide would induce frequency shift in the silicon carbide surface resonance and provide required asymmetry for thermal rectification. We developed a process for plasma enhanced chemical vapor deposition (PECVD) deposition of $\mathrm{SiC}$ and showed that high temperature annealed plasma deposited microcrystalline $\mathrm{SiC}$ exhibits phonon resonance at that same frequency as expected for single crystal silicon carbide. However, the plasma resonance of our microcrystalline $\mathrm{SiC}$ was found to be three times more damped than its single crystal counterpart. Further, simulations were performed to determine the influence of this damping on the rectification behavior.

In the third and fourth part of the project, we performed detailed simulations for heat rectification between nano-beams made of microcrystalline $\mathrm{SiC}$. We also presented an upgraded design of our mechanical platform that is better suited for near-field rectification experiment. The initial design had several limitations like mechanical asymmetries, material stress, lower operating temperature, and broad surface plasmon polarition resonance due to silica coating on nano-beams. These limitations had to be rectified in order to achieve $<50 \mathrm{~nm}$ gap, avoid performance confusion between thermal rectification and mechanical asymmetries, allow higher temperature capabilities and eventually have efficient thermal rectification. The design, after required rectification, is fabricated using our developed polycrystalline silicon carbide. The fabrication processes are modified to reduce stress bending issues. With the new platform we demonstrate near-field radiative heat transfer that is $82 \mathrm{X}$ stronger than the far field limit.

(Milestones achieved: II.1, II.2, and II.3)

In the last part of the project, measurements for thermal rectification are performed on the new platform having silicon nitride nano-beams coated with thin layer of silicon carbide. Poor rectification is observed and simulations using the experimentally obtained damping rate of surface plasmon resonance for $\mathrm{SiC}$ confirm this inefficiency. The simulations show that the rectification for silicon carbide nano-beams is just 7\%. Afterwards, we have performed detailed simulations to explore various material platforms and geometries to achieve proposed rectifications efficiency. Simulations show promising results with alumina, silica, and platinum coated silicon nitride nano-beams having $66-80 \%$ rectification. This proposed platform requires minimal change in our fabrication procedure developed during the entire project. Alumina,

Approved for public release; distribution unlimited. 
silica, and platinum can be deposited on our already fabricated silicon nitride nano-beams coated silicon carbide to perform this experiment. (Milestones achieved: III.1 and III.2) 


\subsection{Technical Details of Main Achievement}

We show strong near-field radiative heat transfer in a novel on-chip geometrical configuration of two parallel suspended nano-beams where the distance between the beams can be tuned electrostatically. Our geometrical configuration is shown in Figure 1a while Figure 1c shows a cross section scheme of a mobile and sensing beam. We use silicon dioxide $\left(\mathrm{SiO}_{2}\right)$ for its surface phonon polariton resonances, at $495 \mathrm{~cm}^{-1}$ and $1160 \mathrm{~cm}^{-1}$, known to allow near-field heat transfer. Silicon nitride $\left(\mathrm{Si}_{3} \mathrm{~N}_{4}\right)$ is used solely for mechanical purposes. We use platinum $(\mathrm{Pt})$ resistors both as resistive heaters and as thermometers to measure the amount of heat transfer.

The gap between the two nano-beams is tuned using a monolithically integrated microelectromechanical system (MEMS) actuator (see Figure 1a). Electrostatic actuation is chosen for its negligible power consumption, and hence negligible parasitic heat generation. When an actuation voltage is applied, the electric field across the two actuation capacitors induces an attraction force that brings the suspended part of the actuator closer to the fixed heated beam (see Figure 1b). When this occurs, the heat transfer between the nano-beams is expected to be drastically enhanced as shown in Figure 1d.
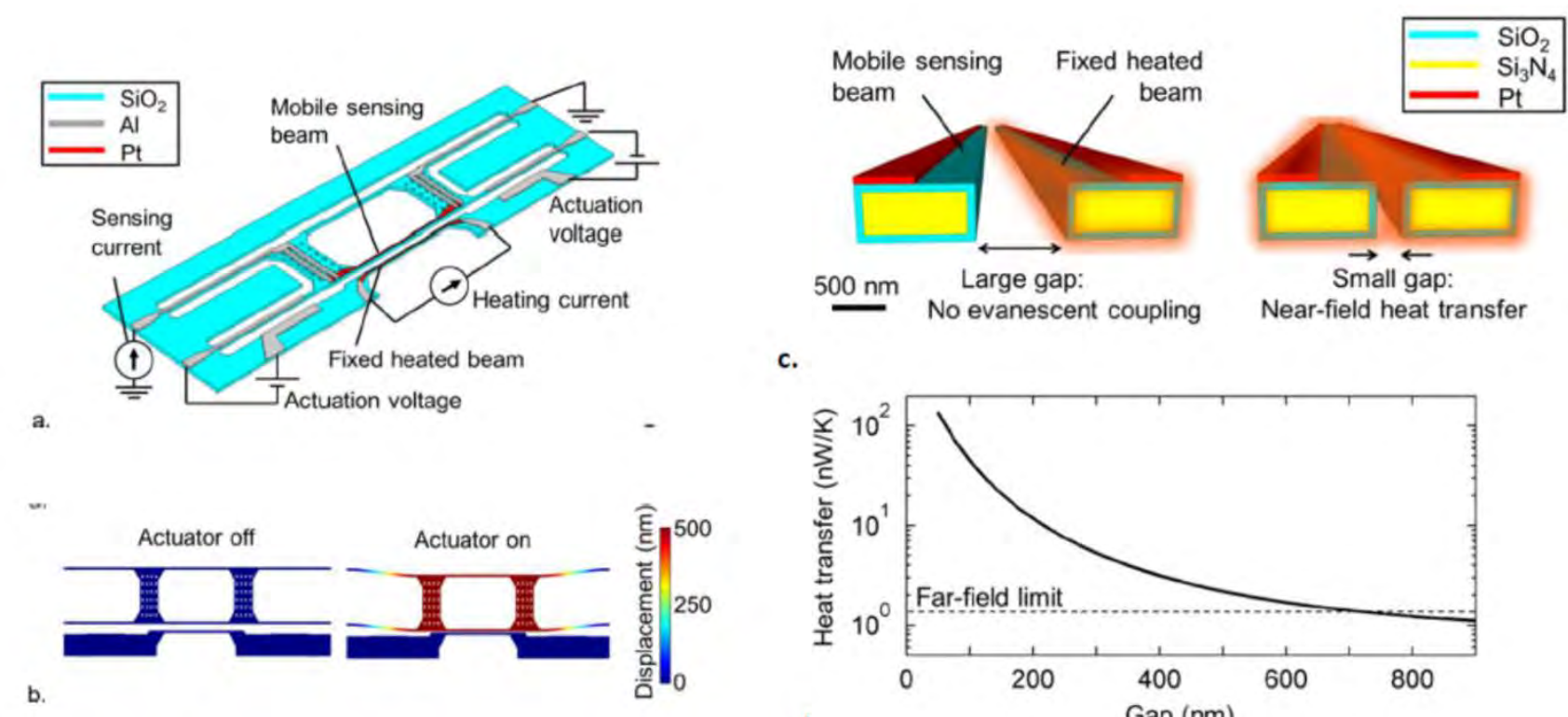

c.

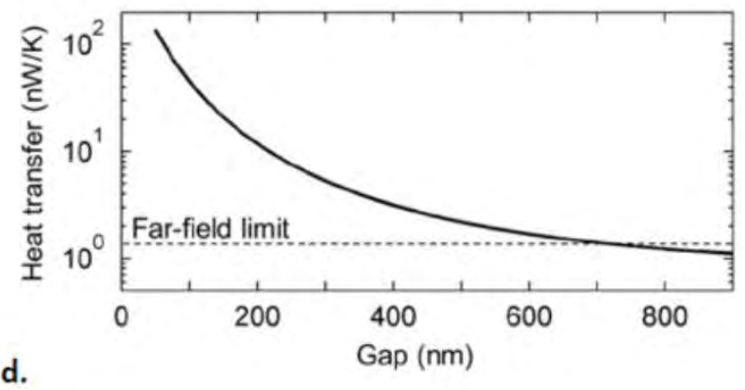

Figure 1: Schematic and Theoretical Prediction

(a) Schematic (not to scale) and electrical circuit of the two nano-beams system integrated with the MEMS actuator, (b) Schematic (not to scale) of the MEMS displacement, (c) Schematic representation of heat transfer between two nano-beams - a fixed and a mobile one. At small gap, evanescent surface polariton resonances at the $\mathrm{SiO}_{2}$ surfaces couple to enable near-field radiative heat transfer between the nano-beams. $\mathrm{Si}_{3} \mathrm{~N}_{4}$ is used for mechanical purposes, while Pt is used both as a resistive heater and a temperature sensor, (d) Theoretical prediction of the heat transfer between two nano-beams of $200 \mu \mathrm{m}$ length, and $500 \mathrm{~nm} \times 1.1 \mu \mathrm{m}$ cross section. The $\mathrm{SiO} 2$ thickness in this case is $100 \mathrm{~nm}$.

Approved for public release; distribution unlimited. 
The structure is fabricated using conventional nanofabrication processes, which consist of low pressure chemical vapor deposition (LPCVD) of $\mathrm{SiO}_{2}$ and $\mathrm{Si}_{3} \mathrm{~N}_{4}$, and electron-beam evaporation of platinum resistors and aluminum electrical contacts. The aluminum layer is chosen to be much thicker $(250 \mathrm{~nm})$ than the platinum layer $(60 \mathrm{~nm})$, such that the resistance of the aluminum contracts is negligible compared with the platinum resistors. The scanning electron micrograph (SEM) images of the device in top view (Figure 2a) and the cross section of the fabricated silicon nitride nano-beams pre-release (Figure $2 b$ ) is shown.

a.

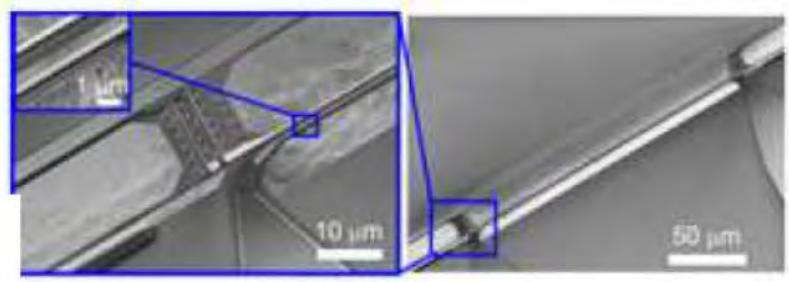

b.

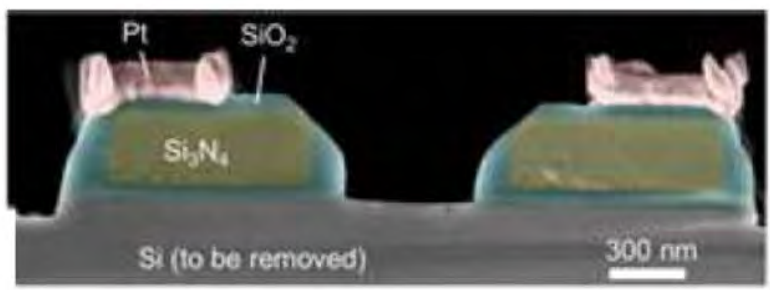

\section{Figure 2: (a) SEMs of the Device and (b) False Color SEM of the Nano-beams Cross Section Prior to Substrate Removal}

The fabricated MEMS platform is found to allow precise control of the nano-beam displacement over $500 \mathrm{~nm}$ with $\pm 10 \mathrm{~nm}$ accuracy. Measurements of temperature changes as a function of nano-beam separation clearly indicate that near-field heat transfer is the dominant thermal transport mechanism between the nano-beams. In the heat transfer experiment, the gap between the nano-beams is progressively reduced using the MEMS actuator, while a constant voltage of $\mathrm{V}_{\mathrm{fix}}=0.22 \mathrm{~V}$ is supplied to heat up the fixed (sensing) nano-beam.

The temperatures of both nano-beams calculated from variation of electrical resistance of platinum resistors as a function of their separation distance is shown in Figure 3a. As the gap decreases, the temperature of the fixed heated beam diminishes slightly as it loses heat to the mobile beam. More importantly, the temperature of the mobile sensing beam increases by almost a factor 5 (from $T_{\text {mob }}=3 \mathrm{~K}$ to $T_{\text {mob }}=14.7 \mathrm{~K}$ ). Such increase is a clear indication that as the gap decreases, near-field effects, rather than conduction through the substrate, dominate heat transfer between the nano-beams. The near-field heat transfer power is extracted from our temperature measurements and is found to be 8 times stronger than substrate conduction, and 7 times stronger than the far-field radiation limit. By solving the thermal circuit of the experiment using the knowledge of background heat conduction of the fixed and mobile beams along with the estimated temperature data from Figure 3a, heat transfer between nano-beams is obtained (see Figure 3b). More details related to the experiment and measurement can be found in our publication based on this work [1-2]. 

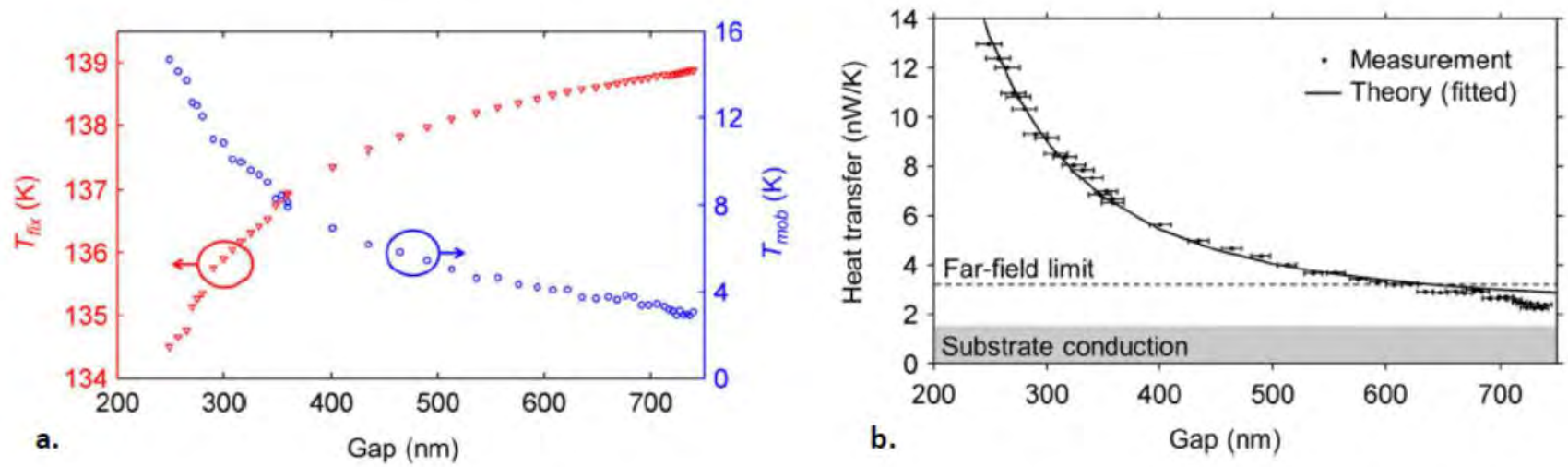

Figure 3: (a) Measured Temperature and (b) Heat Transfer Power

(a) Measured temperature (relative to room temperature) of the fixed heated beam $\left(T_{f i x}\right)$ and the mobile sensing beam $\left(T_{\text {mob }}\right)$ as a function of their separation distance with constant heating voltage supplied to the fixed beam and sensing current to the mobile beam. (b) Heat transfer power between the nano-beams as a function of their separation distance. Substrate conduction is found to account for less than $15 \%$ of the total heat transfer at the smallest gap.

In order to achieve efficient rectification, we need to have a material with narrower surface plasmon resonance. Silicon carbide has been proposed in various theoretical work as a good candidate for thermal rectification platform [3]. Simulations were carried out to understand if silicon carbide nano-beams would allow radiative heat transfer rectification in near-field. For achieving thermal rectification it was found that having silica layer on one of the nano-beam would induce the frequency shift in silicon carbide resonance and ensure the required asymmetry. Thermal rectification mechanism due to misaligned surface plasmon resonance is illustrated schematically in Figure 4.

Approved for public release; distribution unlimited. 
Initial state:

Materials with slightly

misaligned surface resonances
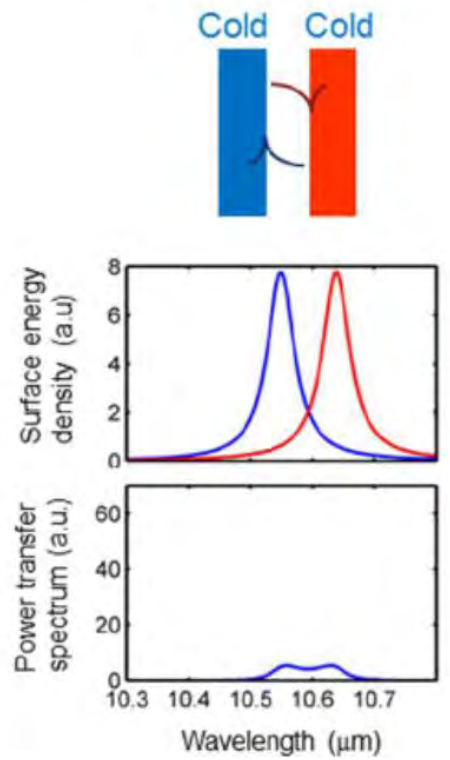

Forward bias:

Surfaces resonances

shift and are aligned
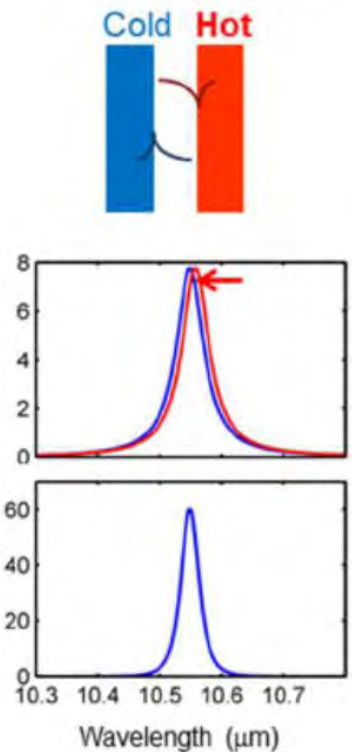

Reverse bias:

Surfaces resonances

further misaligned
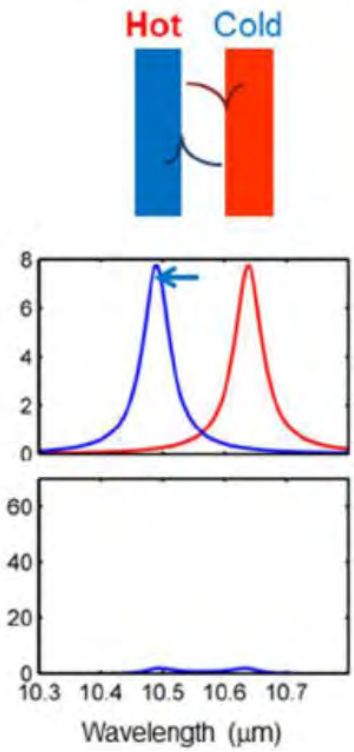

Figure 4: Schematic Representation of the Operation Principle of a Thermal Rectifier Based on misaligned surface waves between two silica coated silicon carbide nano-beams. Different oxide coating thicknesses allow for the initial misalignment between the surface waves.

In order to be able to fabricate a structure with silicon carbide, we had to develop our expertise for its deposition. We developed a process for PECVD of SiC from silane $\left(\mathrm{SiH}_{4}\right)$ and methane $\left(\mathrm{CH}_{4}\right)$ gas precursors, followed by annealing at $1050{ }^{\circ} \mathrm{C}$ for 90 minutes. Systematic optimization for refractive index of deposited films was carried out as a function of silane percentage in the mixture. It is found that $4.5 \%$ silane in the mixture is the optimum point to achieve minimum refractive index and a stoichiometric film. Away from the optimum point, the refractive index increases for excess silicon and carbon. Refer to earlier quarterly report for more details.

We also measured the phonon resonance of our films by recording their infrared transmission spectrum at 70 degree angle of incidence using a Fourier transform infrared spectrometer. As shown in Figure 5a, our film exhibits the same main narrow phonon resonances bands than reported in the literature for single crystal silicon carbide. The experimental peaks are however about three times wider than the theoretical ones. This broadening is most likely a damping effect caused by phonon scattering at the grain boundaries of the microcrystalline film. From this measured phonon resonance, permittivity of our deposited films is extracted which is then used to predict the surface thermal energy spectrum. We have found that surface energy density our deposited $\mathrm{SiC}$ is five times narrower than that of silica which could be beneficial to nearfield thermal rectification (refer to Figure 5b). 

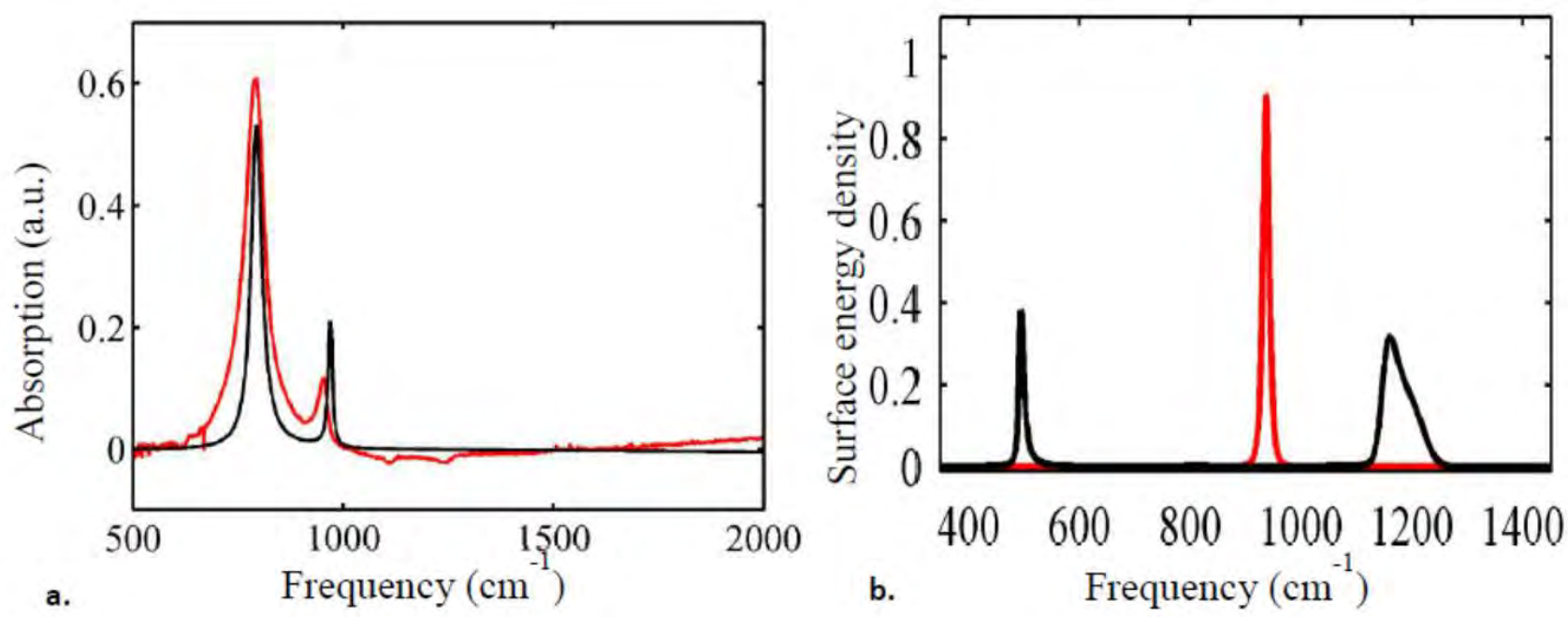

Figure 5: (a) Measurement of the Phonon Resonances of $\mathrm{SiC}$ and Comparison with Theory and (b) Predicted Surface Energy Density of our Deposited SiC Film, Compared to that of $\mathrm{SiO}_{2}$, which was the Material used in our Previous Platform

Simulation studies were undertaken to see thermal rectification between silicon carbide nanobeams coated with silica and the effect of resonance damping as seen in measured plasmon resonance. The simulations performed by Sanford, using full 3D finite element modelling show that ideal silica thickness for rectification (at $100 \mathrm{~nm}$ separation) is $5 \mathrm{~nm}$, for which we expect $20 \%$ rectification. The rectification is found to be linear with temperature, such that a higher temperature bias would allow the targeted $40 \%$ rectification. It is observed in the simulations that having silica films thinner than optimum thickness would lead to insufficient shift in surface resonance while, thicker films would lead to attenuation of surface resonance of coated silicon carbide beam. Either of which is detrimental for thermal rectification.

In order to perform the rectification experiment with silicon carbide nano-beams coated with thin film of silica we had to improve our mechanical platform presented earlier in the quarterly report. In the earlier described platform, the minimum gap achieved was $250 \mathrm{~nm}$, while $\leq 100$ $\mathrm{nm}$ of gap is required to achieve our milestone II.1. The platform also needs to be perfectly symmetric in order to avoid any non-symmetrical effect that could lead to false rectification signal. We also need to achieve temperature as high as $600 \mathrm{~K}$ to perform $40 \%$ rectification. This is not possible with the current design of platinum resistors having aluminum contact pads that limit higher than $400 \mathrm{~K}$ operating temperatures.

The new platform design now provides solution to all these issues. The platinum-resistors are now made more resistive by improved geometry and five times wider contacts to reduce contract resistance. The stress induced deformation (see Figure 8 in first quarterly report), is reduced by having perfectly straight and parallel beams to balance material tensile stress which would prevent deformation upon structural release. Also, the nano-beams are now exactly identical with each having a MEMS actuator as opposed to our initial asymmetric design of having a fixed and a mobile beam (see Figure 6). This would save us from measurement contamination due to asymmetrical effects leading to false conclusions.

Approved for public release; distribution unlimited. 


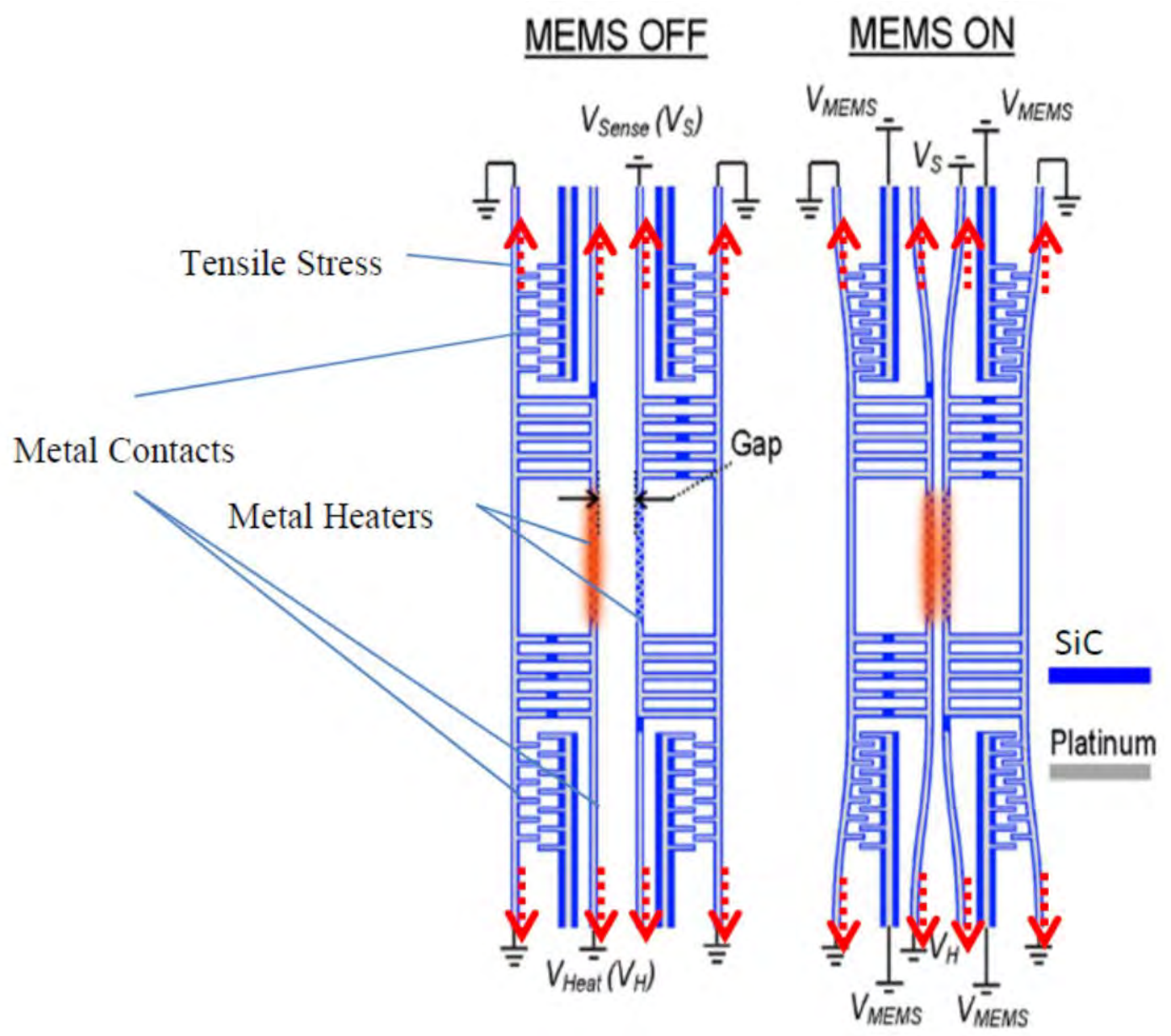

Figure 6: Improved Mechanical Design for Near-field Rectification Measurement The beams are now perfectly parallel to avoid contract points that prevented closing the gap below $250 \mathrm{~nm}$ with the original platform (see Figure 8 in first quarterly report). The system is also perfectly symmetric, in order to avoid spurious false rectification signals from asymmetries

in the system. Platinum is now the only metal used, such that we should achieve higher temperature than with the previous platform (first quarterly report), which included aluminum.

We proceeded to the first fabrication attempt of the new structure design presented above in Figure 6. At first, a thin film microcrystalline silicon carbide $(130 \mathrm{~nm})$ is deposited on a silicon wafer with our developed process followed by annealing and then patterning in fluorine chemistry. The platinum electrical circuitry is then made on top using chrome as adhesion layer. Finally, a $5 \mathrm{~nm}$ thin silica layer is deposited on one of the nano-beams to enable rectification capability.

Although we could successfully fabricate and release the structure (Figure 7a), we found that our developed silicon carbide was not good enough for the intended mechanical use. Our silicon carbide film thickness is limited to $<150 \mathrm{~nm}$ due to inherent stress making thicker films prone to cracking. At lesser thickness, the structure is not stiff enough and consequently suffers from bending. This bending is believed to be caused by a strong stress gradient within the film. The

Approved for public release; distribution unlimited. 
bending causes an out-of-plane displacement of the comb drives teeth (see Figure 7b) which are consequently not interdigitated anymore and unable to actuate the structure.
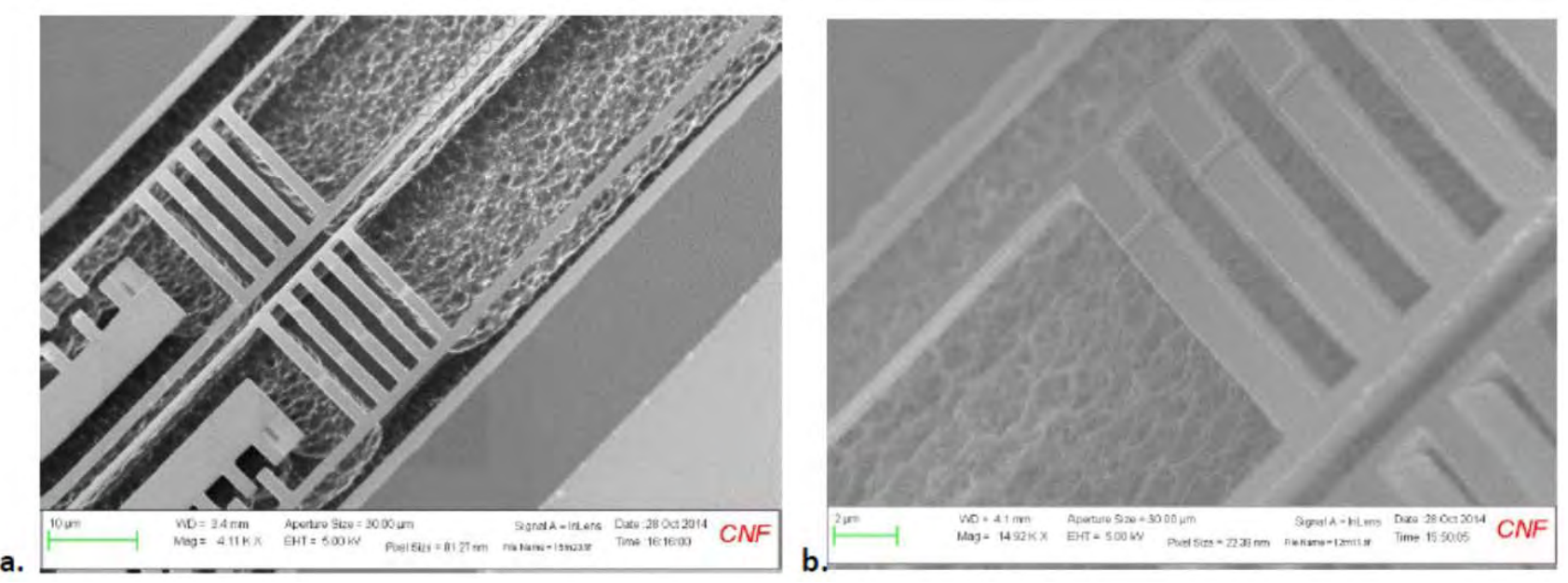

Figure 7: SEM Showing Detrimental Bending of the Device when Fabricated in 130 nm Thick Silicon Carbide

To circumvent this problem, we further modified our design and fabrication process. We now use a mechanical structure made of silicon nitride on which a silicon carbide coating is applied (Figure 8). The fabricated structure shown in Figure 8 is $350 \mathrm{~nm}$ thick and roughly 20 times stiffer than previous structure in silicon carbide. The stiffness of material scales up as (thickness) $^{3}$. Although our system now includes other materials, we expect the heat transfer to be mediated mainly by silicon carbide which constitutes the inner part of the nano-beams.
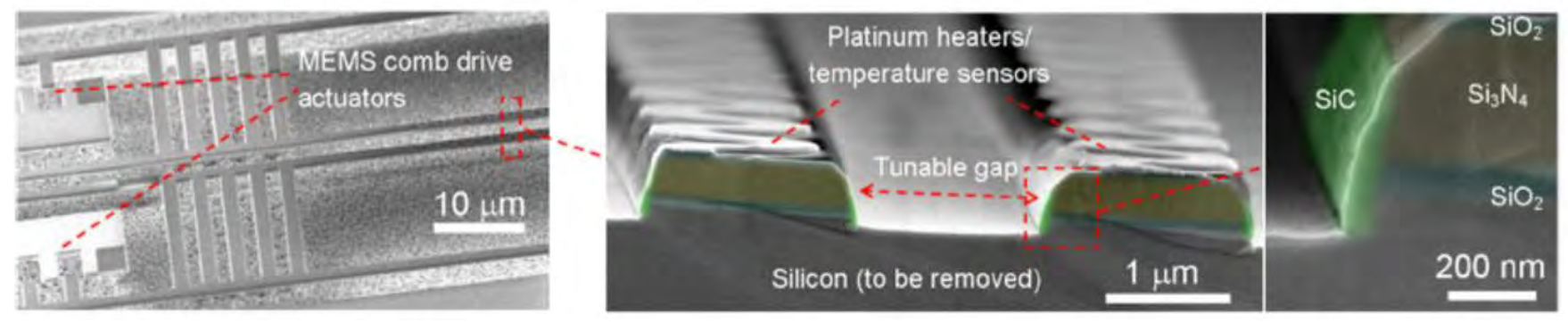

Figure 8: $\mathrm{SEM}$ of the Fabricated Device Based on a $\mathrm{Si}_{3} \mathrm{~N}_{4}$ Mechanical Support Coated with $\mathrm{SiC}$

(Rather than $\mathrm{SiC}$ only in the previous quarterly report)

We find that the mechanical actuation mechanism functions well on this newer implementation of the device, as opposed to the structure that suffered from stress bending in the previous quarterly reports. We measure the MEMS displacement as a function of the voltage applied on the MEMS actuator. This measurement is presented in Figure 9, where we see that the actuator displacement is able to cover the full gap $(1500 \mathrm{~nm})$ between the two structures. The displacement is measured by optical microscopy and by the image treatment algorithm explained in first quarterly report.

Approved for public release; distribution unlimited. 


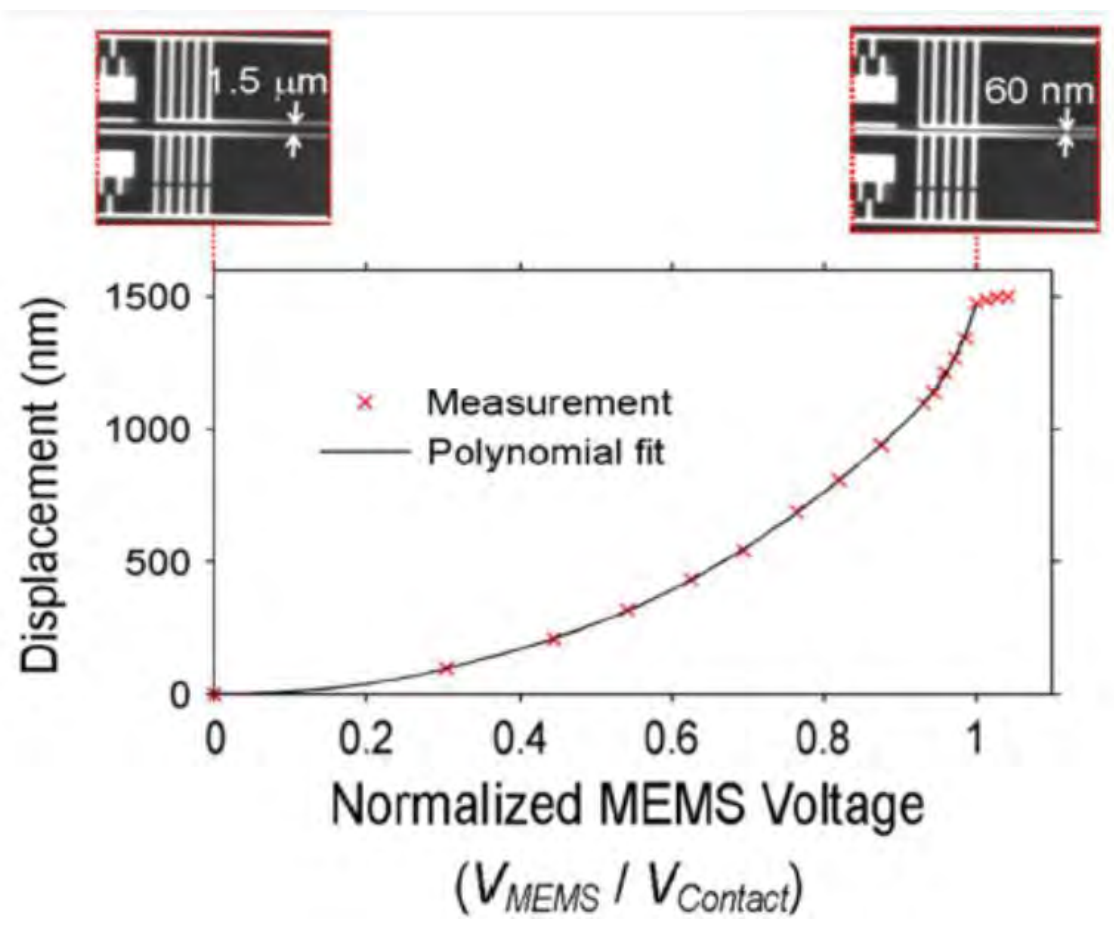

Figure 9: Measured MEMS Displacement as a Function of the Voltage on the MEMS Actuators

We achieve 82X enhancement of heat transfer compared with the far-field limit (Figure 10a). Moreover, we are able to maintain an enhancement greater than $20 \mathrm{X}$ for temperatures as high as $725 \mathrm{~K}$ (Figure 10b). These results meet milestone II.1 amply in term of enhancement. Moreover, the very high temperature achieved will be fundamental to achieving milestone III.3 since, as indicated in the third quarterly report, we expect to need a temperature greater than $600 \mathrm{~K}$ to reach this milestone. The work towards these milestones is reported in our recent publication [4-5].

Heat transfer experiments are performed at room temperature in a high vacuum $\left(9 \times 10^{-5}\right.$ torr $)$ electrical probe stations. The MEMS voltage (VMems) is swept to bring the two nano-beams together while constant heating $\left(\mathrm{V}_{\text {Heat }}\right)$ and sensing $\left(\mathrm{V}_{\text {sens }}\right)$ voltages are supplied to each of the two nano-beams. The time interval between each MEMS voltage increment $(50 \mathrm{~ms})$ is several times larger than the thermal response time of the system $(7 \mathrm{~ms})$, such that all measurements are in steady state. $V_{\text {Sens }}$ is kept much lower than $V_{\text {Heat }}$ such that the power supplied to the sensing

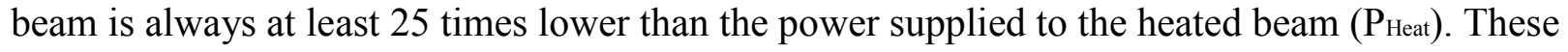
constant voltages are also used to measure the temperature of the two beams, through the variation their electrical resistance (R) as $\Delta \mathrm{R} / \mathrm{R}=\mathrm{TCR} \times \Delta \mathrm{T}$, where $\mathrm{TCR}=0.00166 \mathrm{~K}^{-1}$ is the measured temperature coefficient of resistance of platinum. The measured temperature data are converted to normalized heat transfer power (q, in W/K) using: 


$$
q=\frac{X P_{\text {heat }} T_{\text {sens }}}{\left(T_{\text {heat }}-T_{\text {sens }}\right)\left(T_{\text {heat }}+X T_{\text {sens }}\right)}
$$

Where, $\mathrm{X}$ is the ratio of the background heat conduction (i.e. the heat conduction between the beams and the environment) of the sensing and heated beams $\left(X=\sigma_{\text {sens }} / \sigma_{\text {heat }}\right)$, and $P_{\text {heat }}$ is the supplied heating power. These results are presented in Figure 10a.
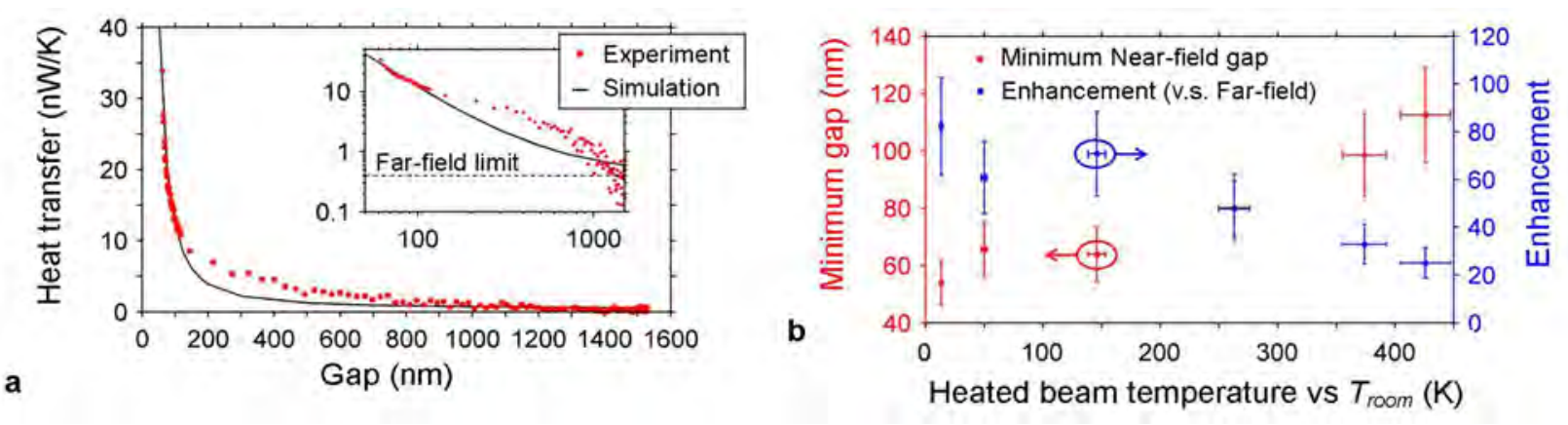

\section{Figure 10: (a) Measurement of Near-field Heat Transfer as a Function of the Distance Between the Nano-beams (the far-field limit is included in the inset (log scale)) and (b) Minimum Achieved Gap (red) and Enhancement over Far-field (blue) as a Function of the Heated Beam Temperature}

Thermal rectification measurements were performed on these devices with silicon nitride nano- beam coated with silicon carbide. There was no rectification seen with these devices. We revisited the thermal rectification simulations this time using the experimentally measured resonance damping of silicon carbide. We found that the surface resonance of our silicon carbide was too damped to have any significant rectification. Grain boundaries seem affect damping coefficient and it seen to be much lower for single crystal $\mathrm{SiC}\left(\Gamma=4.76-8.5 \mathrm{~cm}^{-1}\right.$ as mentioned in literature) while measured to be much higher for polycrystalline silicon carbide $(\Gamma$ $=16.9 \mathrm{~cm}^{-1}$ for our case $)$. Figure 11 shows the rectification coefficient $\left(\left(\mathrm{q}_{\text {forward }}-\mathrm{q}_{\mathrm{r} \text { reverse }}\right) / \mathrm{q}_{\text {reverse}}\right.$, where $\mathrm{q}$ is heat transfer) as obtained using two different values for damping coefficient for the structure shown in Figure 8 with nano-beams having temperature difference of $200 \mathrm{~K}$ and $300 \mathrm{~K}$. As it can be seen in the plot, for our silicon carbide film, having thickness $<100 \mathrm{~nm}$ shows a rectification of just $\sim 7 \%$. 


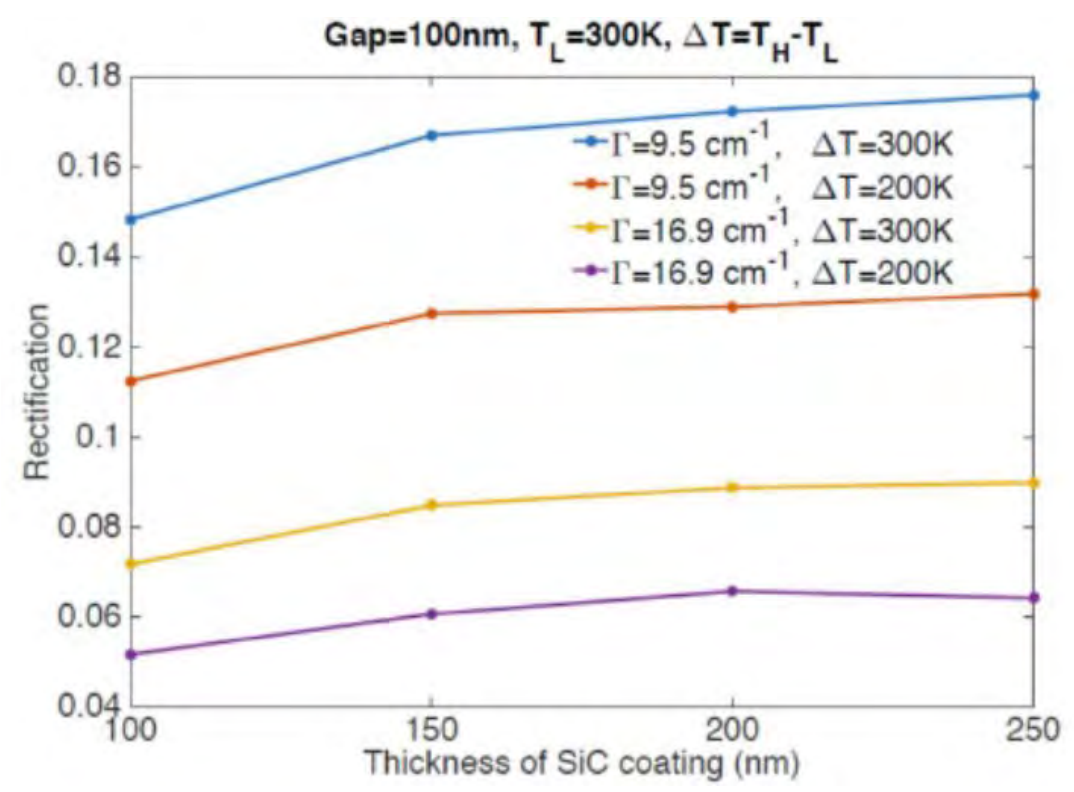

Figure 11: Normalized Rectification as a Function of Film Thickness for Measured Damping Coefficient of our Polycrystalline $\operatorname{SiC}\left(\Gamma=9.5 \mathrm{~cm}^{-1}\right)$

As a comparison, the plot with lower damping coefficient $\left(\Gamma=9.5 \mathrm{~cm}^{-1}\right)$ is also plotted, to show effect of reduced damping on rectification.

The design was revisited in the last part of the project to explore new materials that would fit our requirement of having lower resonance damping, narrower surface resonance, temperature dependent permittivity, better mechanical stability and offering higher operating temperatures. Currently, the known materials that have quite large temperature dependence is $\mathrm{SiC}$, however our experimental data indicates that using polycrystalline $\mathrm{SiC}$ is not good for rectification due to its higher resonance damping. Other materials include $\mathrm{VO}_{2}$ which is incompatible with current fabrication processes and metals which had not been explored until now and are known to show temperature dependence [6]. Also to target a near field rectification which requires heat flow larger than $\mathrm{n}^{2}$ we need to use polar materials like $\mathrm{SiO}_{2}, \mathrm{Si}_{3} \mathrm{~N}_{4}$, doped $\mathrm{Si}, \mathrm{Al}_{2} \mathrm{O}_{3}$ etc., some of which have very little T-dependence (e.g. $\mathrm{SiO}_{2}$ ) and hence unsuitable for our application.

Simulations were done to compute heat transfer between $\mathrm{SiC}$ and metal which showed considerable improvement in rectification. Some of the combinations that showed promising numbers of thermal rectification coefficient with thin metal film are SiC-Au (1.15), SiC-Pd (1.19), SiC-Ni (1.03), and SiC- Pt (0.92). However, it was found that part of heat transfer in these systems was due to non-resonance part of $\mathrm{SiC}$ at wavelengths where it is weakly lossy. This would require us to significantly modify our device dimensions to ensure total absorption of waves and hence heat transfer. A better approach to use $\mathrm{Pt}$ and $\mathrm{SiO}_{2}$ films where the magnitude of heat transfer is seen to be about 2 times higher than $\mathrm{SiC}$ and Pt. Also, it is found that magnitude of transfer power is higher for thinner metal films at resonance as compared to bulk metal (see Figure 12). 


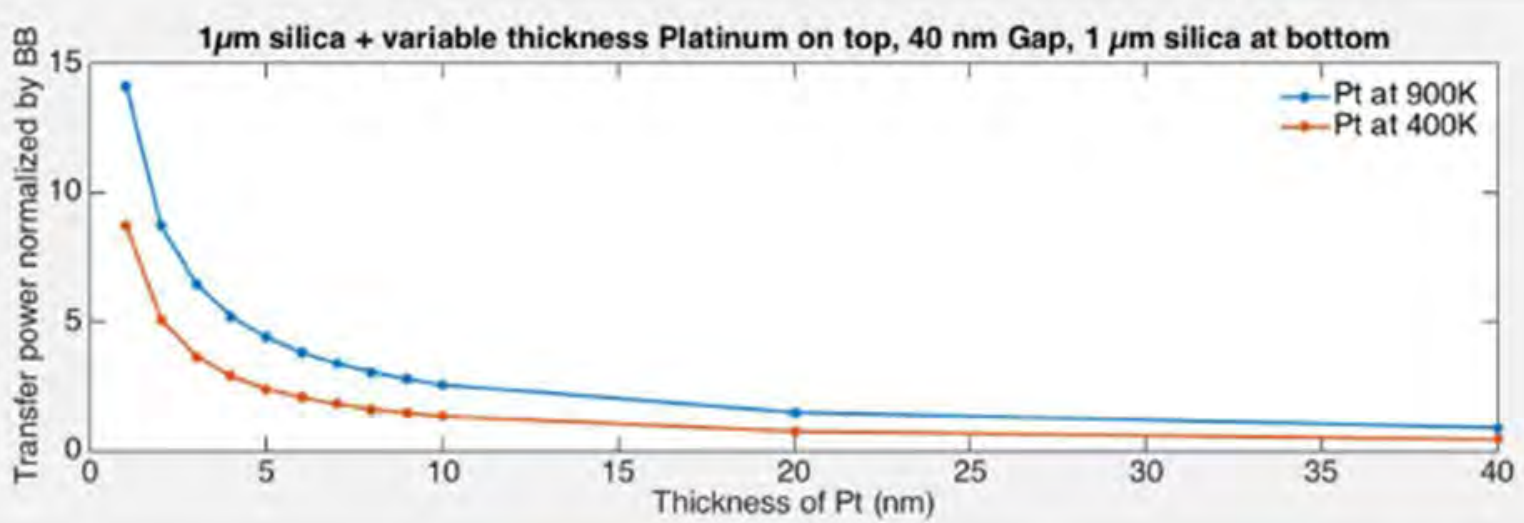

Figure 12: Heat Transfer between Thin $\mathrm{Pt}$ and $\mathrm{SiO}_{2}$ as a Function of Pt Film Thickness for Two Different Temperatures and Normalized by Black Body

We verified the performance between a thin film of another material (alumina) with similar properties (as silica) and metal $(\mathrm{Pt})$ which showed promising results. Finally, we have come up with a design of emitter (nano-beam) that consists of heat transfer between thin film of alumina/silica and $\mathrm{Pt}$ ( $2 \mathrm{~nm}$ ). Figure 13a shows a typical layer stack for our current SiC coated nano-beam after coating them with alumina, silica and platinum. In order to have minimum changes in our current fabrication procedure we propose to coat one of our $\mathrm{Si}_{3} \mathrm{~N}_{4}$ nano-beams with alumina and silica while the other be coated with $\mathrm{Pt}$ thin film $(2 \mathrm{~nm})$. This current step is a simple fix to already fabricated structures having SiC films, where we plan to deposit thin films by placing the chip at an angle. Figure $13 \mathrm{~b}$ is a representational image of our proposed angled evaporation process. The normalized heat transfer plot (Figure 14a), heat transfer spectrum (Figure 14b) and, rectification coefficient (Figure 14c) for the given layer stack are presented below. Rectification coefficient is given as $\left(\mathrm{q}_{\text {forward }}-\mathrm{q}_{\text {reverse }}\right) / \mathrm{q}_{\text {reverse}}$, where $\mathrm{q}$ is heat transfer. We have observed that alumina + silica coated nano-beam showed slightly better performance than having just one of the two material. Enhancement over black body with thin platinum film (2 $\mathrm{nm}$ ) is seen to be maximum (11.2 times), which is seen to reduce (5.8 times) for higher thickness $(10 \mathrm{~nm})$.

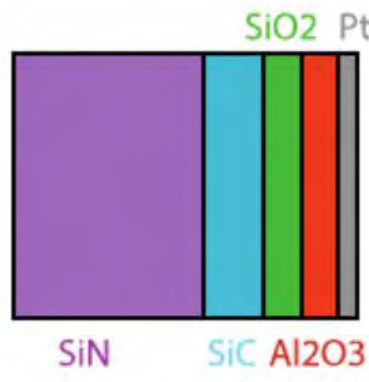

(a)
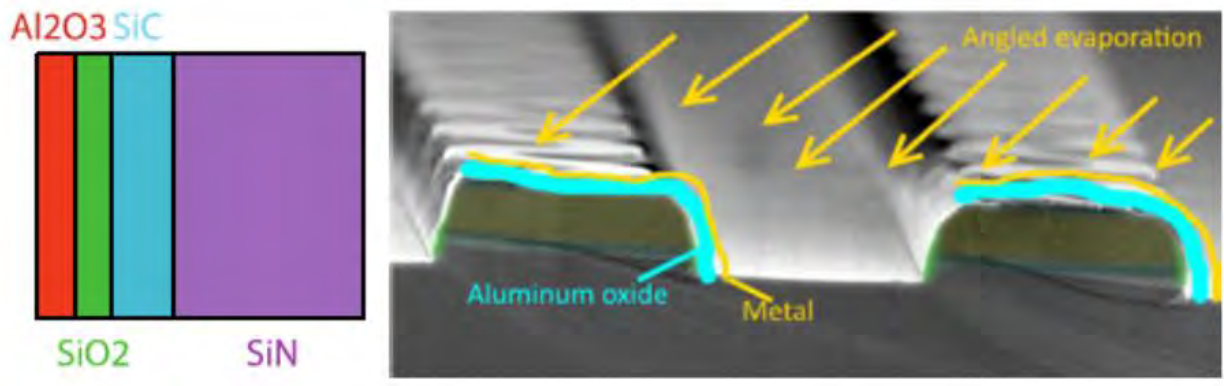

(b)

Figure 13: (a) Multilayer Stack of Materials on Two Nano-beam Surfaces for Efficient Heat Transfer and (b) Angled Evaporation of Currently Fabricated Nano-beams to allow the Required Multilayer Stack on the Inner Heat Exchanging Surfaces

The forward to return heat transfer contrast is $65.7 \%$. 

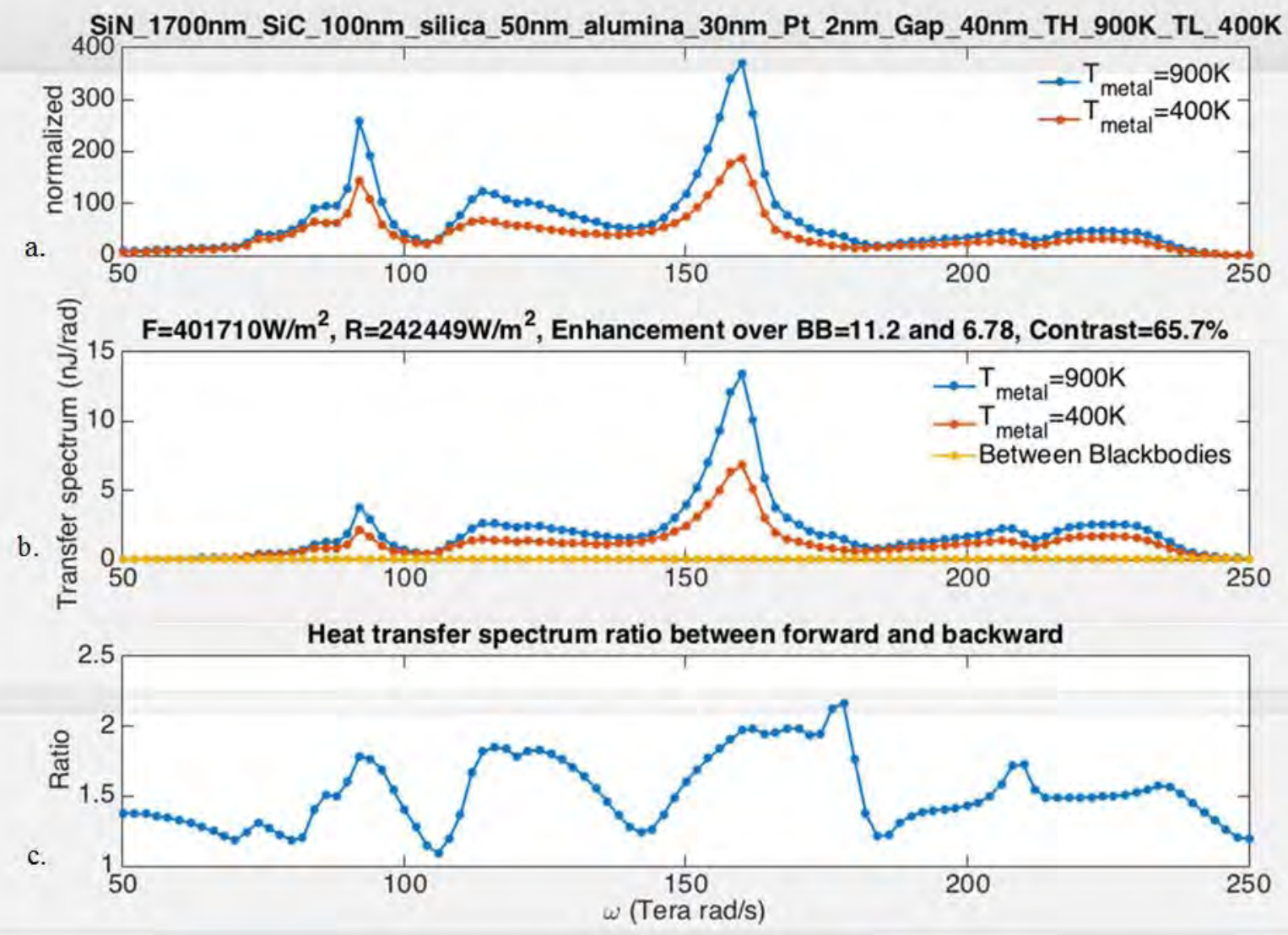

Figure 14: Heat Transfer Spectrum for the Structure Shown in Figure 12a with 40nm Gap

(a) Normalized heat transfer spectrum for two different temperatures, (b) Comparison of heat transfer with black body, and (c) Forward to return heat transfer ratio.

Approved for public release; distribution unlimited. 


\subsection{Milestones Achieved During the Project}

\section{Months}

I.1 Design nano-membranes to exhibit thermal background conductance $<200 \mathrm{nW} / \mathrm{K}$

Done, we have achieved $107 \mathrm{nW} / \mathrm{K}$ experimentally

I.2 Fabricate nano-membranes

Done, see experimental results

I.3 Demonstrate background heat conduction of suspended nano-membranes $<200 \mathrm{nW} / \mathrm{K}$

Done, we have achieved $107 \mathrm{nW} / \mathrm{K}$ experimentally

I.4 Demonstration of suspended nano-membranes thermally isolated from the environment with $<200 \mathrm{nW} / \mathrm{K}$ background conductance, and position controlled $<20 \mathrm{~nm}$ precision

Done, we achieved $10.5 \mathrm{~nm}$ precision positioning experimentally (see discussion related to Figure 5)

\section{Months}

II.1 Demonstration of near-field heat transfer 20 times stronger than the far-field limit

Done, $84 \mathrm{X}$ enhancement at low temperature and $>20 \mathrm{X}$ for temperatures up to $725 \mathrm{~K}$

II.2 Determine the expected rectification dependence with gap between Silicon Carbide nano-beams

Done, gap below $100 \mathrm{~nm}$ is needed to achieve $>40 \%$ rectification together with milestone II.1

II.3 Optimize the thickness of a thin $\mathrm{SiO}_{2}$ coating one of the Silicon Carbide nano-beams to maximize rectification

Done, $5 \mathrm{~nm}$ is the ideal thickness for $100 \mathrm{~nm}$ gap

\section{Months}

III.1 Determine the fundamental limits to radiative rectification of the current platform based on the experimental results (Stanford)

Done, no rectification was seen with the current platform due to high damping of resonance from $\mathrm{SiC}$ 


\section{2 Design of a platform capable of surpassing these limits}

Done, simulation with alumina, silica and platinum coated nano-beams show $\sim 65 \%$ rectification. The current design is slightly modified and we propose to coat one of the two nano-beam with platinum while the other with alumina and silica.

\section{III.3 Demonstration of thermal rectification $>40 \%$ between the two nano-membranes}

Not achieved but potential avenues identified. The current platform, with slight modifications in the deposited layers on the nano-beam surface, is suitable to obtain required separation for near field heat transfer and eventually thermal rectification of $>40 \%$. 


\subsection{References}

[1] Lipson et al., CLEO: 2014 Post deadline Paper Digest, OSA Technical Digest (online), paper FTh5A.8.

[2] Lipson et al., Nano Letters, v. 14, p. 6971 (2014).

[3] Fan et al., J. Appl. Phys. 112, 024304 (2012).

[4] Lipson et al., CLEO: 2015, OSA Technical Digest (online), paper FTh4E.1.

[5] Lipson et al., arXiv:1509.08005 (2015).

[6] Boltasseva et al., Adv. Mater., 25: 3264 (2013). 


\section{Appendix: Publications}

Approved for public release; distribution unlimited. 


\title{
Demonstration of Strong Near-Field Radiative Heat Transfer between Nanostructures
}

\author{
Raphael St-Gelais, ${ }^{1}$ Biswajeet Guha, ${ }^{1}$ Linxiao $\mathrm{Zhu},{ }^{2}$ Shanhui Fan, ${ }^{2}$ and Michal Lipson*,1,3 \\ ${ }^{I}$ School of Electrical and Computer Engineering, Cornell University, Ithaca, New York 14853, \\ United States \\ ${ }^{2}$ Ginzton Laboratory, Stanford University, Stanford, California 94305, United States \\ ${ }^{3}$ Kavli Institute at Cornell for Nanoscience, Cornell University, Ithaca, New York 14853, United States \\ *e-mail:michal.lipson@cornell.edu
}

\begin{abstract}
We demonstrate near-field radiative heat transfer between nanostructures and show that it dominates over other on-chip conduction channels. The measured heat transfer behavior matches the predictions of boundary element method simulations for parallel nanobeams.

OCIS codes: (240.5420) Polaritons; (290.6815) Thermal Emission; (350.4238) Nanophotonics and photonic crystals.
\end{abstract}

Recently, there has been a growing interest in controlling radiative heat flow in the near-field [1], for applications in energy generation (e.g., thermophotovoltaics) and energy extraction (e.g., refrigeration mechanisms). Near-field heat transfer occurs when objects supporting surface phonon-polaritons (e.g., $\mathrm{SiO}_{2}$, $\mathrm{SiC}$ ) or infrared plasmon-polaritons (e.g., doped silicon) are brought to submicron separation, such that their surface modes can evanescently couple and transfer heat with a magnitude that can significantly exceed the blackbody limit.

It has been shown theoretically that near-field thermal control can enable extreme functionalities including thermal transistors and thermal diodes, however these thermal elements would only be relevant to actual systems if shown to occur in integrated nanostructures with arbitrary geometries - where other conduction channels might dominate - rather than in semi-infinite or spherical geometries. To date, nearfield radiative heat transfer has only been demonstrated using macroscopic objects (i.e., one or two semiinfinite surfaces [1-4] or a large probe tip approximated to a sphere [5]).

Here we demonstrate strong near-field radiative heat transfer between two integrated micromechanical nanostructures with novel geometries, and show that the heat transfer can be strong enough to dominate over other conduction channels, even on a fully integrated platform. The nanostructures are composed of two suspended $\mathrm{SiO}_{2}$ coated nanobeams (Fig. 1 a). The suspension mechanism is designed to minimize their background heat conduction, and to enable tuning of their separation distance using a microelectromechanical (MEMS) actuator (Fig. 1 b, c).
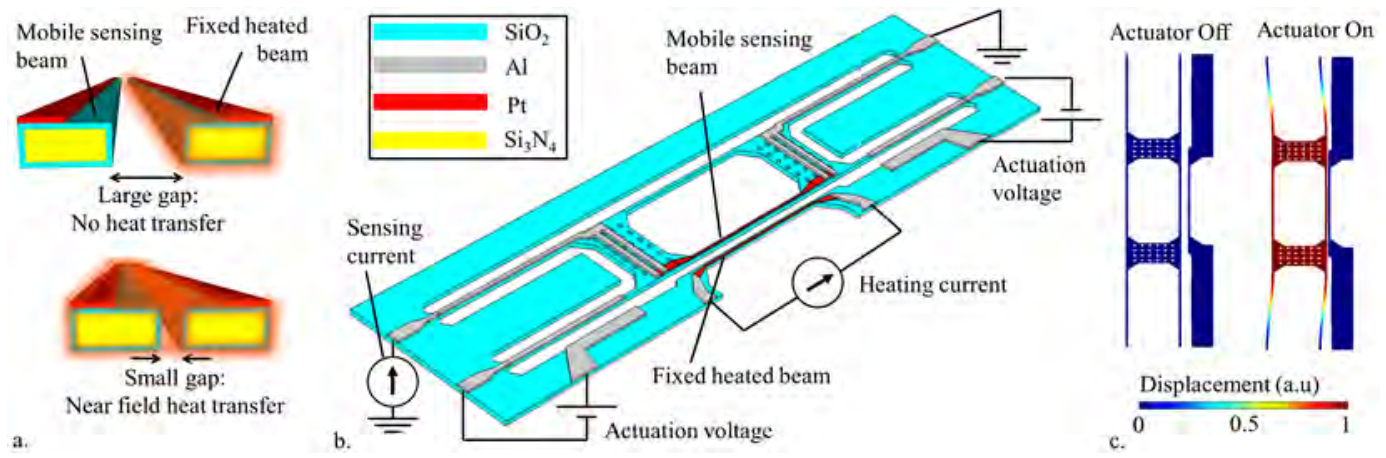

The structure is fabricated using chemical vapor deposition of the layers forming the beams, and e-beam evaporation of the metals forming the heater, sensor and actuator. $\mathrm{XeF}_{2}$ etching is used to undercut the silicon substrate and release the structure. $\mathrm{Si}_{3} \mathrm{~N}_{4}$ is used for mechanical purposes as its tensile residual stress $(\sim 950$ MPa) allow for very long suspended structures [6] (200 $\mu \mathrm{m}$ for the two nanobeams and the four MEMS suspension springs). $\mathrm{SiO}_{2}$ is used for its previously demonstrated ability to support surface-phonon polaritons, and hence to allow near-field heat transfer. $\mathrm{Al}(250 \mathrm{~nm})$ contacts are chosen to be thicker than the 
Pt resistors $(80 \mathrm{~nm})$, such that the overall electrical resistance is dominated by $\operatorname{Pt}(\sim 800 \Omega)$. The fabricated structure is presented in Fig. 2.

Our results clearly indicate that, as the gap between the nanobeams reduces; near-field heat transfer becomes dominant, overcoming any parasitic substrate conduction by more than a factor of four. During the experiment, constant heating current $\left(1.67 \times 10^{-4} \mathrm{~A}\right)$ and sensing current $\left(10^{-6} \mathrm{~A}\right)$ are applied respectively to the fixed (heater) and mobile (sensor) beams. As the gap is tuned, changes of the electrical resistance of the Pt resistors are converted to temperature using a $0.0039 \mathrm{~K}^{-1}$ temperature coefficient of resistance for Pt. A separate experiment was also used to calibrate the temperature of the sensing beams for the initial $555 \mathrm{~nm}$ gap. The temperature of the heater beam relative to room temperature $\left(\Delta \mathrm{T}_{\text {heater }}\right)$ was ramped from 0 to $100 \mathrm{~K}$, while the temperature of the sensing beam $\left(\Delta \mathrm{T}_{\text {sensor }}\right)$ was measured. This calibration data was used to fix the initial values of $\Delta \mathrm{T}_{\text {sensor }}=2 \mathrm{~K}$ and $\Delta \mathrm{T}_{\text {heater }}=75.5 \mathrm{~K}$ in Fig. 3 (a). One can see in Fig. 3 (a) that as the gap decreases from $555 \mathrm{~nm}$ to $100 \mathrm{~nm}, \Delta \mathrm{T}_{\text {sensor }}$ increases by more than a factor of four from 2 to $9 \mathrm{~K}$. This strong gap dependence indicates that the heat conduction between the beams is dominated by near-field effects rather than parasitic background conduction (which are not gap dependent).
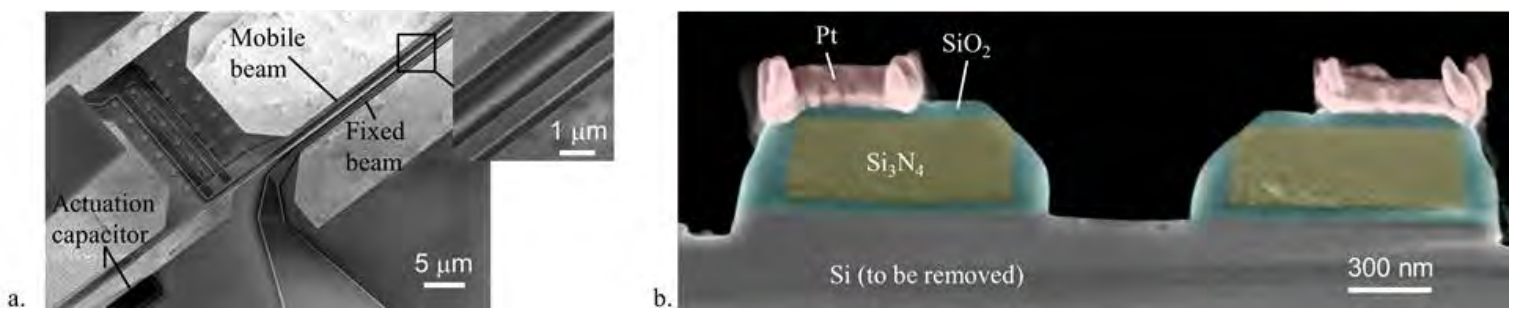

Fig. 2. (a) Scanning electron micrographs of a released device. (b) False color cross section of two nanobeams prior to substrate removal.

We show that in this novel geometry, the heat transfer is inversely proportional to the distance $(d)$ between the nanobeams - which matches well with the $1 / d^{1.68}$ exponential behavior predicted by boundary element method (BEM) simulations. Using conventional equivalent thermal circuit models and the background conductances of the fixed $(400 \mathrm{nW} / \mathrm{K})$ and mobile beam $(235 \mathrm{nW} / \mathrm{K})$, the heat transfer coefficient between the nanobeams $(\sigma)$ is extracted from the heater temperature data of Fig. 3 (a). In Fig. 3 (b), this heat transfer coefficient is fitted to a $\sigma=A / d^{\alpha}+C$ relation where $A$ and $C$ are the fit parameters and $\alpha=1.68$ is fixed. The constant $C$ is included in the fit to account for background (Bgnd) heat conduction through the substrate and yields $C=4.0 \mathrm{nW} / \mathrm{K}$. The $\alpha=1.68$ exponent is obtained by BEM simulations where the nanobeams cross sections can be approximated either as rectangles or as $250 \mathrm{~nm}$ radius cylinders with no noticeable difference (for gaps between 50 and $625 \mathrm{~nm}$ ). The fit corresponds well with the experimental results considering a $\pm 15 \mathrm{~nm}$ uncertainty on the initial gap between the nanobeams (included as error bars in Fig 3 b).
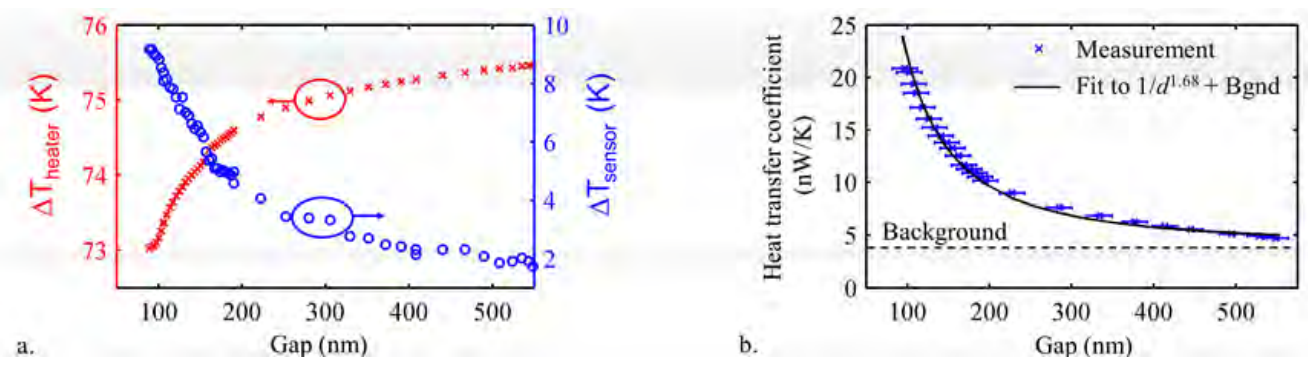

Fig. 3. (a) Temperature (relative to room temperature $\sim 293 \mathrm{~K}$ ) of the mobile sensor beam $\left(\Delta \mathrm{T}_{\text {sensor }}\right)$ and the fixed heater beam

$\left(\Delta \mathrm{T}_{\text {heater }}\right)$ as a function of the gap between them. (b) Heat transfer coefficient between the nanobeams as a function of gap.

We presented the first demonstration of near-field radiative heat transfer between two nanoscale objects and showed that, even when integrated on the same chip, this heat transport mechanism can dominate over other heat conduction channels. We expect our results to have important implications in the development of practical applications of near-field heat transfer, such as thermal rectifiers and switches. 
1. E. Rousseau, A. Siria, G. Jourdan, S. Volz, F. Comin, J. Chevrier, and J.-J. Greffet, "Radiative heat transfer at the nanoscale," Nat Photon 3, 514-517 (2009).

2. A. Kittel, W. Müller-Hirsch, J. Parisi, S.-A. Biehs, D. Reddig, and M. Holthaus, "Near-field Heat transfer in a scanning thermal microscope," Phys Rev Lett 95, 224301 (2005).

3. S. Shen, A. Narayanaswamy, and G. Chen, "Surface phonon polaritons mediated energy transfer between nanoscale gaps," Nano Lett 9, 2909-2913 (2009).

4. R. S. Ottens, V. Quetschke, S. Wise, A. A. Alemi, R. Lundock, G. Mueller, D. H. Reitze, D. B. Tanner, and B. F. Whiting, "Near-field radiative heat transfer between macroscopic planar surfaces," Phys Rev Lett 107, 014301 (2011).

5. B. Guha, C. Otey, C. B. Poitras, S. Fan, and M. Lipson, "Near-field radiative cooling of nanostructures," Nano Lett 12, $4546-4550$ (2012).

6. M. Poot, and H. X. Tang, "Broadband nanoelectromechanical phase shifting of light on a chip," Appl Phys Lett 104, - (2014). 


\title{
Near-Field Radiative Heat Transfer between Integrated Nanostructures using Silicon Carbide
}

\author{
Raphael St-Gelais, ${ }^{1}$ Linxiao Zhu, ${ }^{2}$ Biswajeet Guha, ${ }^{1}$ Shanhui Fan, ${ }^{2}$ and Michal Lipson ${ }^{*, 1,3}$ \\ ${ }^{I}$ School of Electrical and Computer Engineering, Cornell University, Ithaca, New York 14853, United States \\ ${ }^{2}$ Ginzton Laboratory, Stanford University, Stanford, California 94305, United States \\ ${ }^{3}$ Kavli Institute at Cornell for Nanoscience, Cornell University, Ithaca, New York 14853, United States \\ *e-mail: michal.lipson@cornell.edu
}

\begin{abstract}
We present the first experimental demonstration near-field radiative heat transfer using silicon carbide. We achieve a $110 \times$ near-field enhancement, relative to the far-field limit, of the radiative heat transfer between integrated nanostructures.

OCIS codes: (240.5420) Polaritons; (290.6815) Thermal Emission; (350.4238) Nanophotonics and photonic crystals.
\end{abstract}

Near-field radiative heat transfer recently attracted growing interest for applications such as thermal control [1, 2] and thermophotovoltaic energy generation [3, 4]. It occurs when objects supporting surface phonon-polaritons (e.g., $\mathrm{SiO}_{2}, \mathrm{SiC}$ ) or infrared plasmon-polaritons are brought to submicron separation, such that their surface modes can evanescently couple. This coupling enables a radiative transfer of heat that can exceed the far-field blackbody limit by several orders of magnitude, while occurring over a relatively narrow frequency range.

To date, most experimental demonstrations of surface phonon mediated near-field heat-transfer relied on $\mathrm{SiO}_{2}$ as a material platform (e.g., [5-7]), which has limited potential applications due to its relatively broad and multifrequency surface resonance spectrum. Silicon carbide, on the other hand, has a single narrowband surface resonance peak, which also present the advantage of varying dynamically as a function of temperature [1]. These features make $\mathrm{SiC}$ a material of choice for the design frequency tailored near-field thermal emitters $[4,8]$, and for thermal control devices such as thermal rectifiers [1].

Here we present the first experimental demonstration near-field radiative heat transfer using silicon carbide. We use plasma-deposited $\mathrm{SiC}$ to cover the side walls of two parallel nanobeams (see Fig 1 a). These nanobeams are integrated on a microelectromechanical (MEMS) platform (Fig. $1 \mathrm{~b}$ ), similar to the one previously reported [7], that allow precise tuning of the distance between the nanobeams and measurement of the heat transfer. Platinum resistors on top of the beams are used both as resistive heaters and as temperature sensors. $\mathrm{Si}_{3} \mathrm{~N}_{4}$ is used for mechanical purposes, as its tensile stress allow for long suspended nanostructures, while $\mathrm{SiO}_{2}$ is used solely as a protective coating for $\mathrm{Si}_{3} \mathrm{~N}_{4}$ during structural release. The fabrication process of the device, except for deposition of silicon carbide, is the same as in [7].

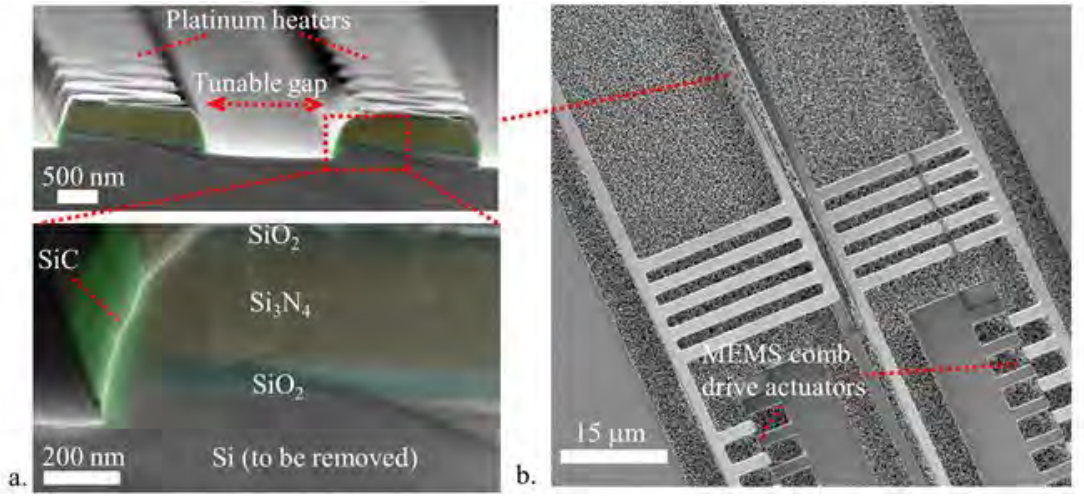

Fig. 1. (a) False color cross-section view of the silicon carbide coated nanobeams prior to structural release. (b) Top view of structurally released nanobeams integrated with the MEMS actuation mechanism.

We characterize plasma deposited silicon carbide and predict a significantly narrower near-field heat transfer spectrum than with the usual $\mathrm{SiO}_{2}$ material platforms. Amorphous $\mathrm{SiC}$ is deposited over the nanobeams by plasma enhanced chemical vapor deposition (PECVD) from $\mathrm{SiH}_{4}$ and $\mathrm{CH}_{4}$ gas precursors. It is then anisotropically etched, such that carbide is present only on the side walls of the nanobeams (see Fig. 1 a). The substrate is subsequently annealed at $1050{ }^{\circ} \mathrm{C}$ for 90 minutes to enable the formation of a microcrystalline SiC phase [9]. The mid-infrared permittivity of annealed $\mathrm{SiC}$ is extracted from the mid-infrared transmission spectrum at $70^{\circ}$ grazing incidence of a 
$120 \mathrm{~nm}$ thick film deposited on a blank silicon wafer. From this measurement, we find that the permittivity of the deposited material is described remarkably well by a Lorentz-Drude dispersion model, $\varepsilon(\omega)=\varepsilon_{\infty}+\varepsilon_{\infty}\left(\omega_{L}^{2}-\omega_{T}^{2}\right) /\left(\omega_{T}^{2}-\omega^{2}-\mathrm{i} \Gamma \omega\right)$, with $\omega_{L}=955 \mathrm{~cm}^{-1}, \omega_{T}=790 \mathrm{~cm}^{-1}, \Gamma=17 \mathrm{~cm}^{-1}$, and $\varepsilon_{\infty}=7.1$. Using this permittivity we expect, in the general case of two parallel plates [10], a $4.5 \times$ narrower near-field heat transfer spectrum than using the permittivity of $\mathrm{SiO}_{2}[11]$. We also expect narrower spectra for virtually any other geometry, although we have not yet investigated specific cases.

Our silicon carbide coating is found to enable strong near-field radiative heat transfer between two integrated nanobeams, showing a $110 \times$ near-field enhancement of heat transfer relative to the far-field limit. During the experiment, a constant $0.4 \mathrm{~V}$ heating voltage is applied to one of the nanobeams, while a constant $50 \mathrm{mV}$ sensing voltage is applied to the other beam. The voltage across the MEMS actuators is then swept from 0 to $140 \mathrm{~V}$ to bring the two nanobeams closer together (see Fig. 2 a), while simultaneously measuring the nanobeams temperatures from the change of their electrical resistance [7]. The normalized radiative power transferred (Fig. $2 \mathrm{c}$ ) is then calculated from the measured nanobeam temperatures (Fig. 2 b). The gap values are obtained from the voltage applied on the MEMS actuator and by fitting the experimental heat transfer data to simulated values. These simulations are obtained by the Fourier modal method [7, 12], for a $145 \mathrm{~K}$ temperature difference between the beams. The simulated values are also fitted along the vertical axis to account for parasitic heat conduction through the substrate $(\sim 2 \mathrm{nW} / \mathrm{K})$. The far-field limit is obtained by simulating the power radiated in the far-field by the silicon carbide surface of one of our nanobeam $(0.79 \mathrm{nW} / \mathrm{K})$, which is considered to be at $\Delta \mathrm{T}=185 \mathrm{~K}$ above room temperature.
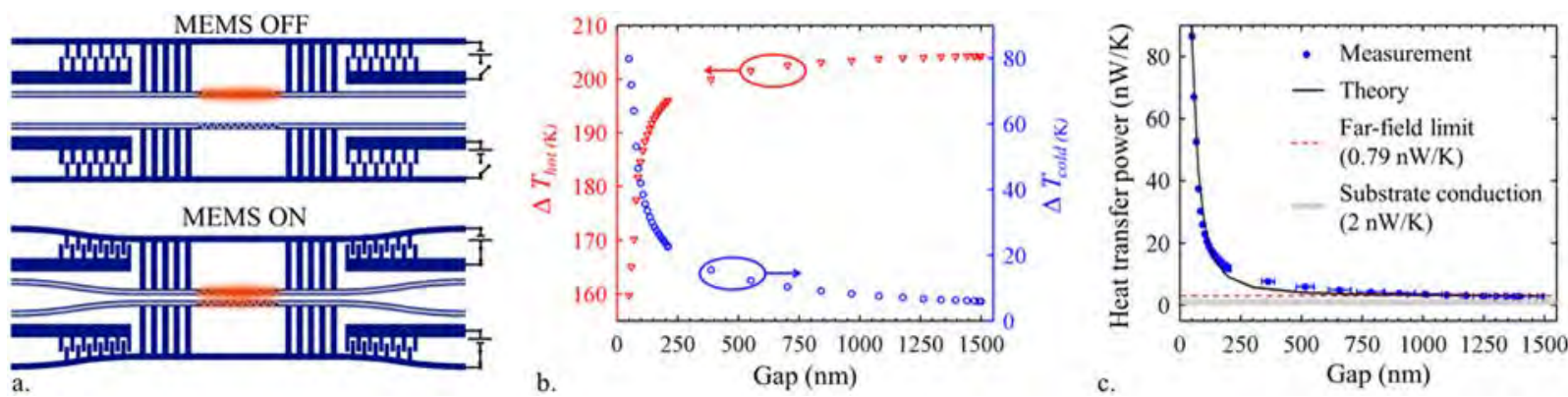

Fig. 2. (a) Schematic representation of the experiment (not to scale). (b) Temperature, relative to ambient, of the heated ( $\Delta T_{h o t}$ ) and the sensing $\left(\Delta T_{\text {cold }}\right)$ beams as a function of their separation distance (c) Normalized heat transfer power between two $200 \mu \mathrm{m}$ long nanobeams as a function of their separation distance.

We showed that plasma-deposited $\mathrm{SiC}$ can enable strong near-field radiative heat transfer. We also achieved a $110 \times$ near-field enhancement of heat transfer, which is unprecedented for integrated nanostructures. These achievements should contribute to the development of thermal control [1] and thermo photovoltaic [4] devices.

1. C. R. Otey, W. T. Lau, and S. Fan, "Thermal Rectification through Vacuum," Phys Rev Lett 104, 154301 (2010).

2. $\quad$ P. Ben-Abdallah and S.-A. Biehs, "Near-Field Thermal Transistor," Phys Rev Lett 112, 044301 (2014).

3. S. Basu, Z. M. Zhang, and C. J. Fu, "Review of near-field thermal radiation and its application to energy conversion," Int J Energ Res 33, 1203-1232 (2009).

4. A. Narayanaswamy and G. Chen, "Surface modes for near field thermophotovoltaics," Applied Physics Letters 82, $3544-3546$ (2003).

5. E. Rousseau, A. Siria, G. Jourdan, S. Volz, F. Comin, J. Chevrier, and J.-J. Greffet, "Radiative heat transfer at the nanoscale," Nat Photon 3, 514-517 (2009).

6. S. Shen, A. Narayanaswamy, and G. Chen, "Surface phonon polaritons mediated energy transfer between nanoscale gaps," Nano Lett 9, 2909-2913 (2009).

7. R. St-Gelais, B. Guha, L. Zhu, S. Fan, and M. Lipson, "Demonstration of Strong Near-Field Radiative Heat Transfer between Integrated Nanostructures," Nano Letters (2014).

8. M. Francoeur, M. Pinar Mengüç, and R. Vaillon, "Spectral tuning of near-field radiative heat flux between two thin silicon carbide films," Journal of Physics D: Applied Physics 43, 075501 (2010).

9. L. Calcagno, P. Musumeci, F. Roccaforte, C. Bongiorno, and G. Foti, "Crystallization process of amorphous silicon-carbon alloys," Thin Solid Films 411, 298-302 (2002).

10. K. Joulain, J.-P. Mulet, F. Marquier, R. Carminati, and J.-J. Greffet, "Surface electromagnetic waves thermally excited: Radiative heat transfer, coherence properties and Casimir forces revisited in the near field," Surface Science Reports 57, 59-112 (2005).

11. R. Kitamura, L. Pilon, and M. Jonasz, "Optical constants of silica glass from extreme ultraviolet to far infrared at near room temperature," Appl. Opt. 46, 8118-8133 (2007).

12. C. R. Otey, L. Zhu, S. Sandhu, and S. Fan, "Fluctuational electrodynamics calculations of near-field heat transfer in non-planar geometries: A brief overview," J Quant Spectrosc RA 132, 3-11 (2014). 


\title{
Demonstration of Strong Near-Field Radiative Heat Transfer between Integrated Nanostructures
}

\author{
Raphael St-Gelais, ${ }^{\dagger}$ Biswajeet Guha, ${ }^{\dagger}$ Linxiao Zhu, ${ }^{\ddagger}$ Shanhui Fan, ${ }^{\ddagger}$ and Michal Lipson ${ }^{*,+\S}$ \\ ${ }^{\dagger}$ School of Electrical and Computer Engineering, Cornell University, Ithaca, New York 14853, United States \\ ${ }^{\ddagger}$ Ginzton Laboratory, Stanford University, Stanford, California 94305, United States \\ ${ }^{\S}$ Kavli Institute at Cornell for Nanoscience, Cornell University, Ithaca, New York 14853, United States \\ S Supporting Information
}

ABSTRACT: Near-field heat transfer recently attracted growing interest but was demonstrated experimentally only in macro- scopic systems. However, several projected applications would be relevant mostly in integrated nanostructures. Here we demon- strate a platform for nearfield heat transfer on-chip and show that it can be the dominant thermal transport mechanism between integrated nanostructures, overcoming background substrate conduction and the far-field limit (by factors 8 and 7, respectively). Our approach could enable the development of active thermal control devices such as thermal rectifiers and transistors.

KEYWORDS: Thermal transport, thermal radiation, near-field radiation, microelectromechanical systems (MEMS),
Recently there has been a growing interest in controlling radiative heat transfer in the near-field, ${ }^{1-11}$ for applications in thermal miscroscopy, ${ }^{12-14}$ thermophotovoltaic energy generation, ${ }^{15-18}$ noncontact cooling, ${ }^{19,20}$ and heat flow control. ${ }^{21-28}$ Near-field heat transfer occurs when objects supporting surface phononpolaritons (e.g., $\mathrm{SiO}_{2}$ and $\mathrm{SiC}$ ) or infrared plasmonpolaritons (e.g., doped silicon) are brought to submicron separation, such that their surface modes can evanescently couple. This heat transfer occurs over a narrow frequency range (as opposed to the broadband nature of solid state conduction) and can exceed the blackbody limit by several orders of magnitude.

It has been shown theoretically that near-field heat transfer can enable active functionalities such as thermal rectifiers, ${ }^{21-23,25-27}$ thermal transistors, ${ }^{28}$ and thermal switches; ${ }^{24}$ however, these devices would only be relevant to actual systems if shown to occur in integrated geometries, where unfortunately other conduction channels might dominate, rather than in macroscopic object. To date, near-field heat transfer has only been shown using macroscopic objects, i.e., one or two semiinfinite surfaces, ${ }^{7,8,10-15,19}$ or a large probe tip approximated as a sphere. ${ }^{20}$ Scaling up these macroscopic geometries to an actual thermal circuit composed of several components would be extremely challenging since even a single thermal transistor requires at least two near-field heat transfer junctions. On-chip integration is therefore necessary for the development of several applications. Moreover, miniaturization could eventually yield fundamental performance advantages over macroscopic experiments. For example, nanopatterned objects can significantly relax the distance requirement for efficient near-field heat transfer between two objects, ${ }^{9}$ while size-induced discretization of thermal modes could allow ultrahigh contrast rectification of heat transfer. ${ }^{27}$ Here we show strong near-field radiative heat transfer in a novel on-chip geometrical configuration of two parallel suspended nanobeams where the distance between the beams can be tuned electrostatically. Our geometrical configuration is shown in Figure 1a. We use silicon dioxide $\left(\mathrm{SiO}_{2}\right)$ for its surface phonon polariton resonances, at 495 and $1160 \mathrm{~cm}^{-1}$, shown to allow nearfield heat transfer. ${ }^{7,8,11,20}$ Silicon nitride $\left(\mathrm{Si}_{3} \mathrm{~N}_{4}\right)$ is used solely for mechanical purposes as its tensile residual stress ( 950 MPA) allows for long suspended nanobeams that are thermally isolated from the substrate. We use platinum $(\mathrm{Pt})$ resistors both as resistive heaters and as thermometers to measure the amount of heat transfer.

The gap between the two nanobeams is tuned using a monolithically integrated microelectromechanical (MEMS) actuator (see Figure 2a). Electrostatic actuation is chosen for its negligible power consumption and hence negligible parasitic heat generation. When an actuation voltage is applied, the electric field across the two actuation capacitors induces an attraction force that brings the suspended part of the actuator closer to the fixed heated beam (see Figure 2b). The design of the MEMS, in particular the use of deposited metal electrodes over a nitride mechanical platform, was inspired in part by ref

Received: August 22, 2014

Revised: October 30, 2014

Published: November 24, 2014 

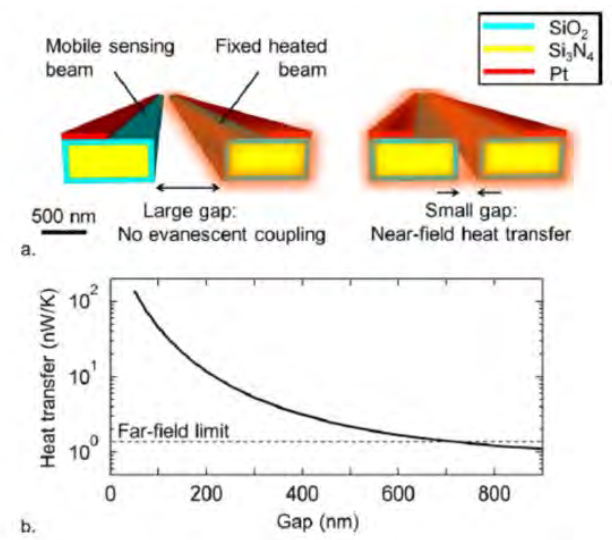

Figure 1. (a) Schematic representation of the experiment. At small gap, evanescent surface polariton resonances at the $\mathrm{SiO}_{2}$ surfaces couple to enable near-field radiative heat transfer between the nanobeams. Si3N4 is used for mechanical purposes, while $\mathrm{Pt}$ is used both as a resistive heater and a temperature sensor. (b) Theoretical prediction of the heat transfer between two nanobeams of $200 \mu \mathrm{m}$ length and $500 \mathrm{~nm} \times 1.1 \mu \mathrm{m}$ cross-section. The $\mathrm{SiO}_{2}$ thickness in this case is $100 \mathrm{~nm}$.

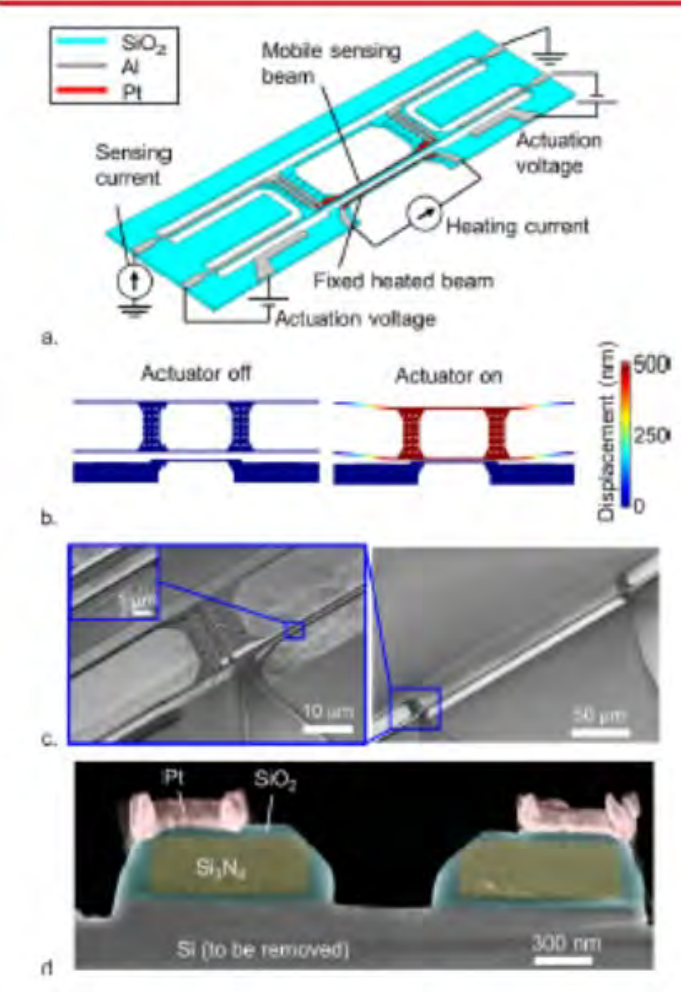

Figure 2. (a) Schematic (not to scale) and electrical circuit of the two- nanobeam system integrated with the MEMS actuator. (b) Schematic (not to scale) of the MEMS displacement. (c) Scanning electron micrographs (SEM) of the device. (d) False color SEM of the nanobeam cross section prior to substrate removal.
29. To tune the gap continuously while avoiding electrostatic pull-in effect, the gap between the electrodes of the actuation capacitors $(3 \mu \mathrm{m})$ is designed to be more than three times larger than the maximum possible displacement (given by the gap between the nanobeams, designed to be $700 \mathrm{~nm}$ ). The length of the fixed beam, the mobile sensing beam, and each of the four MEMS suspension springs is $200 \mu \mathrm{m}$. Such large dimensions are required to minimize the mechanical stiffness of the suspension springs and hence to allow full range displacement with an actuation voltage below $200 \mathrm{~V}$. These large dimensions are also beneficial to the thermal isolation of the nanobeams (i.e., to the minimization of the background thermal heat conduction between the nanobeams and the substrate).

The simulated heat transfer power between the nanobeams presents a drastic enhancement of heat transfer in the near-field (see Figure 1b). The near-field simulation in Figure $1 \mathrm{~b}$ is performed using a Fourier modal method based on the fluctuational electrodynamics formalism, ${ }^{30,31}$ considering beams of $200 \mu \mathrm{m}$ length and $500 \mathrm{~nm} \times 1.1 \mu \mathrm{m}$ cross section (including a $100 \mathrm{~nm}$ thick $\mathrm{SiO}_{2}$ coating). The temperature difference between the nanobeams is $130 \mathrm{~K}$ (as in our experimental results), with the mobile sensing beam maintained at room temperature. Repeating the near-field simulation while replacing the $\mathrm{Si}_{3} \mathrm{~N}_{4}$ core with $\mathrm{SiO}_{2}$ shows that the $\mathrm{Si}_{3} \mathrm{~N}_{4}$ core has a negligible effect on the heat transfer compared with a beam that would be made entirely of $\mathrm{SiO}_{2}$. The insensitivity of heat transfer to the core material results from the surface wave nature of the $\mathrm{SiO}_{2}$ surface phononpolariton that dominates heat transfer at small gaps. In the gap range of Figure 1b, the heat transfer power approximately scales as $1 /$ gap $^{1.68}$. At much smaller distances (i.e., distances much smaller than the beam dimensions), we expect the heat transfer to be proportional to $1 /$ gap $^{2}$, as for parallel plates. ${ }^{19}$ The farfield value in Figure 1b is the total far-field emission, integrated over all directions and all frequencies, for a nanobeam maintained at $130 \mathrm{~K}$ above room temperature. This value is calculated by the Fourier modal analysis method for a periodic array of nanobeams with a periodicity much larger than the size of nanobeams, such that they do not interact with each other.

The structure is fabricated using conventional nanofabrication processes, which consist of low pressure chemical vapor deposition (LPCVD) of $\mathrm{SiO}_{2}$ and $\mathrm{Si}_{3} \mathrm{~N}_{4}$, and electron- beam evaporation of platinum resistors and aluminum electrical contacts. The fabrication process begins with the successive deposition of $100 \mathrm{~nm}$ of $\mathrm{SiO}_{2}$, $300 \mathrm{~nm}$ of $\mathrm{Si}_{3} \mathrm{~N}_{4}$, and $100 \mathrm{~nm}$ of $\mathrm{SiO}_{2}$ on a virgin silicon wafer. The MEMS and nanobeams are then defined by deep ultraviolet lithography and etched in $\mathrm{CHF}_{3}+\mathrm{O}_{2}$ chemistry using an inductively coupled plasma reactive ion etching (ICP-RIE) reactor. Following this etch step, a third layer of $\mathrm{SiO}_{2}$ is deposited, again by LPCVD, in order to conformally cover the sidewalls of the etched structures. This layer is then anisotropically etched (using the same ICP-RIE chemistry) to clear the bottom of the 
trenches for subsequent isotropic release, while leaving some $\mathrm{SiO} 2$ on the sidewalls of the nanobeams (see the final nanobeams cross section in Figure 2d). Platinum and aluminum are then successively deposited over the defined structure by electron beam evaporation and liftoff. The aluminum layer is chosen to be much thicker $(250 \mathrm{~nm})$ than the platinum layer $(60 \mathrm{~nm})$, such that the resistance of the aluminum contacts is negligible compared with the platinum resistors. The higher electrical conductivity of aluminum, relative to platinum, also contributes to make the resistance of the aluminum contacts negligible. The structure is finally released in $\mathrm{XeF}_{2}$ gas isotropic etching of silicon. Release holes are included on the larger parts of the MEMS (see Figure 2c) to facilitate the isotropic release step.

The fabricated MEMS platform is found to allow precise control of the nanobeam displacement over $500 \mathrm{~nm}$ with $\pm 10 \mathrm{~nm}$ accuracy. The displacement of the MEMS actuator as a function of the applied voltage is measured using a custom- made image processing algorithm coupled with optical microscopy. Optical images of the MEMS are taken at a 50× magnification (see Figure $3 \mathrm{a}$ ) for different actuation voltages.

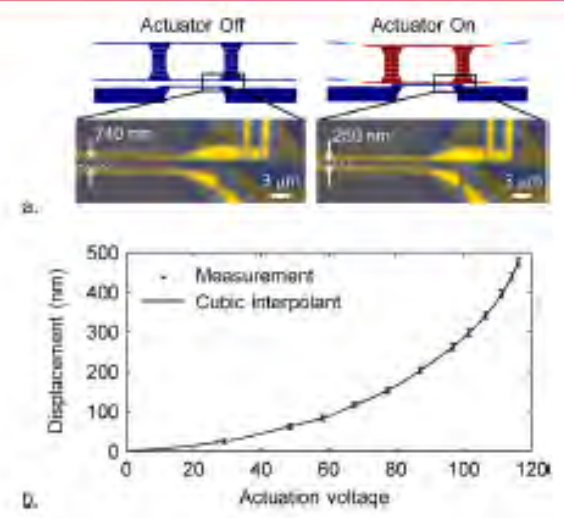

Figure 3. (a) Optical images of the MEMS displacement at actuation voltages of 0 (left) and $116 \mathrm{~V}$ (right). The images are treated by our image processing algorithm to extract the MEMS displacement. (b) Measured MEMS displacement as a function of the actuation voltage. The cubic spline interpolation is used to convert actuation voltages to displacement values.

The relative position of the two beams is then extracted by fitting Gaussian distributions over the nanobeam images. This fitting algorithm is repeated and averaged over all cross-section slices of each image file (i.e., over each vertical slice of the nanobeams in Figure 3a). The measured displacement of the mobile nanobeam is presented in Figure 3b, which shows smooth and continuous control of the MEMS position as a function of the applied voltage. The uncertainty on the measured displacement arises from the precision of the image processing algorithm $( \pm 5.5 \mathrm{~nm})$ and from the uncertainty on the scale of the image $(67.8 \pm 0.7 \mathrm{~nm} / \mathrm{pixel})$. The detailed calculation of these uncertainty values is presented in Supporting Information S1. It should be noted that, although we can measure the relative displacement of the MEMS with great accuracy, our knowledge of the absolute distance between the two nanobeams suffers from greater uncertainty due to our difficulty in evaluating the initial beam separation distance $\left(d_{0}=740 \mathrm{~nm}\right)$. Although $d_{0}$ is fixed by design, it changes significantly upon release due to internal material stress and must therefore be evaluated from scanning electron micrographs of the released device. We also note that the maximum achieved displacement in Figure $3 \mathrm{~b}$ is slightly below $500 \mathrm{~nm}$, while the initial gap is around 740 $\mathrm{nm}$, which means that the minimum achievable gap is in the $200-250 \mathrm{~nm}$ range. The reason why the gap cannot be reduced further is not fundamental and is detailed in Supporting Information S2.

Measurements of temperature changes as a function of nanobeam separation clearly indicate that near-field heat transfer is the dominant thermal transport mechanism between the nanobeams. All heat transfer experiments are carried in vacuum at a pressure of $1.5 \times 10^{-4}$ Torr. The electrical measurements are performed using an Agilent B1500A semiconductor device parameter analyzer. In the heat transfer experiment, the gap between the nanobeams is progressively reduced using the MEMS actuator, while a constant voltage of $V_{\text {fix }}=0.22 \mathrm{~V}$ is supplied to heat up the fixed nanobeam (which translates to $33 \mu \mathrm{W}$ of electrical power at the initial beam separation). Meanwhile, the temperature of the mobile sensing beam is measured using a $10 \mu \mathrm{A}$ sensing current (which translates to $0.12 \mu \mathrm{W}$ electrical power, more than 2 orders of magnitude lower than the heating power applied to the fixed beam). To ensure that the system is in its steady state at each measurement point, a $0.2 \mathrm{~s}$ delay is included between the MEMS actuation voltage setting and the temperature readout. This delay is orders of magnitude larger than the thermal time constants of the fixed heated beam $(1.3 \mathrm{~ms})$ and the mobile sensing beam $(7.4 \mathrm{~ms})$. Those time constants were measured in a separated experiment by applying a step voltage variation to the nanobeam resistors while measuring their transient current. The temperatures of both nanobeams are calculated from the measured electrical resistance $(R)$ of their platinum resistors, which changes as $\triangle R / R=T C R \times$ $\Delta T$, where the temperature coefficient of resistance $(T C R)$ is $0.00181 \mathrm{~K}^{-1}$. The $T C R$ value was calibrated separately by measuring the resistance of a device placed on a hot plate ramped from 20 to $150{ }^{\circ} \mathrm{C}$. The temperature (relative to room temperature) of both the fixed heated beam and the mobile sensing beam, as a function of their separation distance, is presented in Figure 4. As the gap decreases, the temperature of the fixed heated beam diminishes slightly as it loses heat to the mobile beam. More importantly, the temperature of the mobile sensing beam increases by almost a factor of 5 (from $T_{\text {mob }}=3 \mathrm{~K}$ to $T_{\mathrm{mob}}=14.7 \mathrm{~K}$ ). Such increase is a clear indication that as the gap decreases, near-field effects, rather than conduction through the substrate, dominate heat transfer between the nanobeams (substrate conduction should indeed be gap independent). 


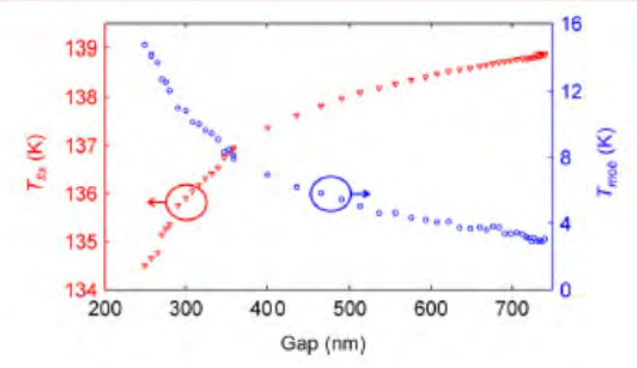

Figure 4. Measured temperature (relative to room temperature) of the fixed heated beam $\left(T_{\text {fix }}\right)$ and the mobile sensing beam $\left(T_{\mathrm{mob}}\right)$ as a function of their separation distance. In this case, a constant heating voltage is supplied to the fixed beam, while only a sensing current is supplied to the mobile beam.

The near-field heat transfer power is extracted from our temperature measurements and is found to be 8 times stronger than substrate conduction and 7 times stronger than the far- field radiation limit. We can convert the temperature data of Figure 4 to heat transfer power values (q) using eq 1, which is obtained by solving the equivalent thermal circuit of the experiment (as is routinely done for on-chip thermal transport experiments $^{32}$ ).

$$
q=\frac{P_{\text {tix }} X T_{\text {mob }}}{\left(T_{\text {fix }}-T_{\text {mob }}\right)\left(T_{\text {fix }}+X T_{\text {mob }}\right)}
$$

In eq $1, q$ is the power transfer (in Watt/K) normalized by the temperature difference between the beams, $T_{\text {fix }}$ and $T_{\text {mob }}$ are, respectively, the temperature of the fixed heated beam and the mobile sensing beam, and $X=\sigma_{\text {mob }} / \sigma_{\text {fix }}$ is the ratio of the background heat conductions of the nanobeams. For each nanobeam, the background heat conduction between the beam and the substrate (i.e., $\sigma_{\text {mob }}$ and $\left.\sigma_{\text {fix }}\right)$ is determined by applying a ramping electrical power to the beam while simultaneously measuring its temperature (i.e., its electrical resistance). The inverse of the slope of this temperature vs electrical power measurement yields the background conduction, in units of Watt $/ \mathrm{K}$. We obtain conduction values of $\sigma_{\text {fix }}=237$ $\mathrm{nW} / \mathrm{K}$ and $\sigma_{\text {mob }}=107 \mathrm{nW} / \mathrm{K}$ for the fixed heated beam and the mobile sensing beam, respectively. Using these values and the temperature data of Figure 4, we obtain the heat transfer power as a function of the nanobeam separation (see Figure 5). In Figure 5, the horizontal error bars correspond to the error on the measurement of the MEMS displacement (see Figure 3). Vertical error bars are not visible, as they are determined by the very high resolution of the Agilent device parameter analyzer. The theory curve is the same as in Figure 1, but is now translated horizontally and vertically to best fit the experimental data. The horizontal translation is included to account from our uncertainty on the initial distance $\left(d_{0}\right)$ between the beams (see discussion related to Figure 3 and the MEMS displacement measurement), while the vertical translation accounts for spurious conduction of heat through the substrate. The translation that best fits the experimental data is $+49 \mathrm{~nm}$ horizontally and +1.49
$\mathrm{nW} / \mathrm{K}$ vertically. After fitting, the theory is found to correspond closely with the experimental data. Slight discrepancies between theory and measurements most likely arise from deviation of the beam cross-section from the perfectly rectangular shape considered in the simulations (see Figure 2d). The $1.49 \mathrm{nW} / \mathrm{K}$ susbtrate conduction obtained from the fit is included in Figure 5, from which we note that spurious substrate conduction account for less than $12 \%$ of the total heat transfer at the smallest gap. The far-field radiation limit $(1.7 \mathrm{nW} / \mathrm{K})$ is also included in Figure 5 in order to illustrate the strong enhancement of heat transfer in the near- field compared to the far-field value. The far-field emission is obtained by the same Fourier modal method used in Figure 1.

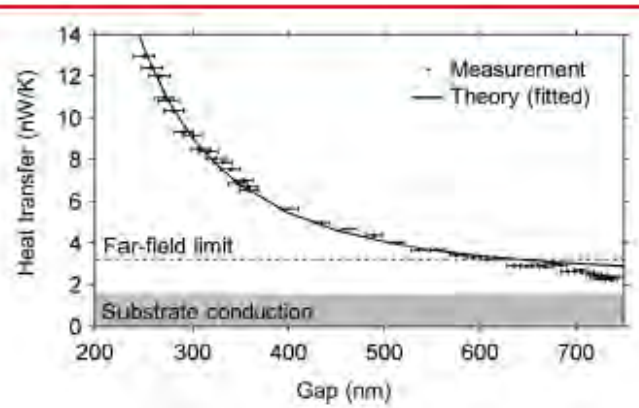

Figure 5. Heat transfer power between the nanobeams as a function of their separation distance. Substrate conduction is found to account for less than $15 \%$ of the total heat transfer at the smallest gap. Near-field heat transfer is also found to be 7 times stronger than the far-field limit $(1.7 \mathrm{nW} / \mathrm{K}$ above the substrate conduction, for the current geometry and temperatures).

We have presented the first demonstration of near-field radiative heat transfer between two integrated nanostructures and shown that near-field radiation can be the dominant heat transport channel between these structures, even on a fully integrated platform. The approach, based on nanobeam integration with MEMS actuation, could enable the development of new near-field thermal control devices, such as thermal rectifiers and thermal transistors.

\section{ASSOCIATED CONTENT}

Supporting Information

(1) Uncertainty on the MEMS displacement

measurement. (2) Discussion on the minimum achievable nanobeam separation. (3) Supplemental calibration of the temperature measurement. This material is available free of charge via the Internet at http://pubs.acs.org.

\section{AUTHOR INFORMATION}

Corresponding Author

*E-mail: michal.lipson@cornell.edu.

Notes

The authors declare no competing financial interest. 


\section{ACKNOWLEDGMENTS}

The authors gratefully acknowledge Clayton Otey for using some of his codes for the calibrations of the numerical simulations presented here. The authors gratefully acknowledge support from DARPA for award FA8650-14-1-7406 supervised by Dr. Avram Bar-Cohen. This work made use of the Cornell Center for Materials Research Shared Facilities, which are supported through the NSF MRSEC program (DMR-1120296), and of the Cornell NanoScale Facility, a member of the National Nanotechnology Infrastructure Network, which is supported by the National Science Foundation (Grant ECCS-0335765). R.S.-G. holds a postdoctoral fellowship from the Fonds de recherche du Quebec-Nature et Technologies.

\section{REFERENCES}

(1) Polder, D.; Van Hove, M. Phys. Rev. B 1971, 4 (10), 3303-3314.

(2) Loomis, J. J.; Maris, H. J. Phys. Rev. B 1994, 50 (24), 18517-18524.

(3) Pendry, J. B. J. Phys.: Condens. Matter 1999, 11 (35), 6621.

(4) Fu, C. J.; Zhang, Z. M. Int. J. Heat Mass Transfer 2006, 49 (9-10), 1703-1718.

(5) Volokitin, A. I.; Persson, B. N. J. Rev. Mod. Phys. 2007, 79 (4), 1291-1329.

(6) Francoeur, M.; Mengüc, M. P.; Vaillon, R. Appl. Phys. Lett. 2008, 93 (4), 043109.

(7) Rousseau, E.; Siria, A.; Jourdan, G.; Volz, S.; Comin, F.; Chevrier, J.; Greffet, J.-J. Nat. Photon. 2009, 3 (9), 514-517.

(8) Shen, S.; Narayanaswamy, A.; Chen, G. Nano Lett. 2009, 9 (8), 2909-2913.

(9) Rodriguez, A. W.; Ilic, O.; Bermel, P.; Celanovic, I.; Joannopoulos, J. D.; Soljacic', 2011, 107 (11), 114302.

M.; Johnson, S. G. Phys. Rev. Lett. 2011, 107 (11),

114302.

(10) Kralik, T.; Hanzelka, P.; Zobac, M.; Musilova, V.;

Fort, T.;Horak, M. Phys. Rev. Lett. 2012, 109 (22),

224302.

(11) Shi, J.; Li, P.; Liu, B.; Shen, S. Appl. Phys. Lett. 2013, 102 (18), 183114.

(12) Kittel, A.; Müller-Hirsch, W.; Parisi, J.; Biehs, S.A.; Reddig, D.; Holthaus, M. Phys. Rev. Lett. 2005, 95 (22), 224301

(13) De Wilde, Y.; Formanek, F.; Carminati, R.; Gralak, B.; Lemoine, P.-A.; Joulain, K.; Mulet, J.-P.; Chen, Y.; Greffet, J.-J. Nature 2006, 444 (7120), 740-743.

(14) Jones, A. C.; Raschke, M. B. Nano Lett. 2012, 12

(3), 1475-1481.

(15) DiMatteo, R. S.; Greiff, P.; Finberg, S. L.; YoungWaithe, K. A.; Choy, H. K. H.; Masaki, M. M.; Fonstad, C. G. Appl. Phys. Lett. 2001, 79 (12), 1894-1896.

(16) DiMatteo, R.; Greiff, P.; Seltzer, D.; Meulenberg, D.; Brown, E.;Carlen, E.; Kaiser, K.; Finberg, S.;

Nguyen, H.; Azarkevich, J. In Micron-gap

Thermophotovoltaics (MTPV), Sixth Conference on
Thermophotovoltaic Generation of Electricity: TPV6; AIP Publishing: Melville, NY, 2004; pp 42-51.

(17) Laroche, M.; Carminati, R.; Greffet, J.-J. J. Appl. Phys. 2006, 100 (6), 063704.

(18) Basu, S.; Zhang, Z. M.; Fu, C. J. Int. J. Energy Res. 2009, 33 (13), 1203-1232.

(19) Ottens, R. S.; Quetschke, V.; Wise, S.; Alemi, A. A.; Lundock, R.; Mueller, G.; Reitze, D. H.; Tanner, D. B.; Whiting, B. F. Phys. Rev. Lett. 2011, 107 (1), 014301. (20) Guha, B.; Otey, C.; Poitras, C. B.; Fan, S.; Lipson, M. Nano Lett. 2012, 12 (9), 4546-4550.

(21) Otey, C. R.; Lau, W. T.; Fan, S. Phys. Rev. Lett. 2010, 104 (15), 154301.

(22) Basu, S.; Francoeur, M. Appl. Phys. Lett. 2011, 98

(11), 113106.

(23) Iizuka, H.; Fan, S. J. Appl. Phys. 2012, 112 (2), 024304.

(24) Zhu, L.; Otey, C. R.; Fan, S. Appl. Phys. Lett. 2012, 100 (4), 044104.

(25) Wang, L. P.; Zhang, Z. M. Nanoscale Microscale Thermophys. Eng. 2013, 17 (4), 337-348.

(26) Park, K.; Zhang, Z. Front. Heat Mass Transfer 2013, 4 (1), 013001.

(27) Zhu, L.; Otey, C. R.; Fan, S. Phys. Rev. B 2013, 88

(18), 184301.

(28) Ben-Abdallah, P.; Biehs, S.-A. Phys. Rev. Lett. 2014, 112 (4), 044301.

(29) Poot, M.; Tang, H. X. Appl. Phys. Lett. 2014, 104

(6), 061101.

(30) Lussange, J.; Guerout, R.; Rosa, F. S. S.; Greffet, J. J.; Lambrecht, A.; Reynaud, S. Phys. Rev. B 2012, 86 (8), 085432.

(31) Otey, C. R.; Zhu, L.; Sandhu, S.; Fan, S. J. Quant. Spectrosc. Radiat. Transfer 2014, 132 (0), 3-11.

(32) Chang, C. W.; Okawa, D.; Majumdar, A.; Zettl, A. Science 2006, 314 (5802), 1121-1124. 


\title{
Near-field radiative heat transfer between nanostructures in the deep sub-wavelength regime
}

\author{
Raphael St-Gelais, ${ }^{1,2}$ Linxiao Zhu, ${ }^{3}$ Shanhui Fan, ${ }^{3}$ and Michal Lipson ${ }^{*}, 1,2$ \\ ${ }^{1}$ School of Electrical and Computer Engineering, Cornell University, Ithaca, New York 14853, United States \\ ${ }^{2}$ Department of Electrical Engineering, Columbia University, New York, New York 10027, United States \\ ${ }^{3}$ Ginzton Laboratory, Stanford University, Stanford, California 94305, United States \\ *e-mail:ml3745@columbia.edu
}

\section{Introductory paragraph}

Radiative heat transfer between parallel objects separated by deep sub-wavelength distances and subject to large thermal gradients $(>100 \mathrm{~K})$ could enable breakthrough technologies for electricity generation $^{1-5}$ and thermal transport control ${ }^{6-8}$. However, thermal transport in this regime has never been achieved experimentally due to the difficulty of maintaining large thermal gradients over nmscale distances while avoiding other heat transfer mechanism such as conduction. Previous experimental measurement between parallel planes ${ }^{1,9-13}$ were limited to distances greater than 500 $\mathrm{nm}^{11}$ (with a $20 \mathrm{~K}$ thermal gradient), which is much larger than the theoretically predicted distance $(<100 \mathrm{~nm})$ required for most applications ${ }^{1-8}$. Here we show near- field radiative heat transfer between parallel nanostructures in the deep sub-wavelength regime using high precision micro electromechanical (MEMS) displacement control. We also exploit the high mechanical stability of structures under high tensile stress to minimize thermal buckling effects and maintain small separations at large thermal gradients. We achieve an enhancement of heat transfer of almost two orders of magnitude relative to the far-field limit, which corresponds to a $54 \mathrm{~nm}$ separation. We also achieve a high temperature gradient $(260 \mathrm{~K})$ between the cold and hot surfaces while maintaining a $\sim 100 \mathrm{~nm}$ distance.

\section{Main text}

Radiative heat transfer between objects separated by deep sub-wavelength distances can exceed the conventional laws of thermal radiation ${ }^{14,15}$, while being concentrated over a quasi- monochromatic frequency range ${ }^{16}$. This heat transfer occurs through evanescent coupling of thermally excited surface resonances, and consequently scales inversely with separation (as $1 / d^{\alpha}$ where $\alpha$ is a geometry dependent factor). The separation $(d)$ at which this regime occurs depends on the geometry of the system and on the materials involved, but it is generally around $200 \mathrm{~nm}$ for identical parallel structures relying on surface phonon resonances ${ }^{17}$. For applications such as electricity generation and thermal control, the two materials involved are non-identical, such that resonant coupling is less efficient and separations $<100 \mathrm{~nm}$ are typically required ${ }^{3,18}$.

The unique features of heat transfer in the deep sub-wavelength regime (i.e., large magnitude and quasi-monochromatic spectral distribution) could allow high efficiency generation of electricity from heat ${ }^{1,3-5}$ and novel thermal control devices ${ }^{6,8}$. For example, near-field heat transfer between a hot thermal emitter and a cold photovoltaic cell could allow energy generation with a greater efficiency ( $>30 \%$ for a $T=600 \mathrm{~K}$ heat source ${ }^{5}$ ) than using either thermoelectric generator or single junction solar photovoltaic cells. It is also predicted that engineering the surface resonance frequencies of parallel structures could allow novel thermal control devices such as thermal rectifiers ${ }^{6,7}$ or thermal transistors ${ }^{8}$. 
These applications rely on radiative heat transfer between parallel structures in the deep subwavelength regime, and on a high temperature gradient between them, neither of which have been achieved experimentally. Near-field enhancement of heat transfer was demonstrated between parallel plates using active parallelism control ${ }^{10,12,13}$, or mechanical spacers ${ }^{1,9,11}$. The smallest separation achieved in these experiments is $500 \mathrm{~nm}^{11}$ (with a $20 \mathrm{~K}$ thermal gradient), which is small enough to overcome the far-field blackbody radiation limit, but not enough to reach the deep sub-wavelength regime where the heat flux is effectively concentrated around a single frequency ${ }^{17}$. Furthermore, the highest temperature gradient $(\Delta T)$ achieved in these experiments is $85 \mathrm{~K}^{9}$ (for a $1.6 \mu \mathrm{m}$ separation), which is relatively small, especially for energy generation applications where the generated power and the efficiency $(\eta)$ both scale with the temperature gradient (e.g., $\eta_{\text {Carnot }}=$ $\left.\Delta T / T_{h o t}\right)$. In sphere-plane geometries ${ }^{19-21}$, distances as low as $20 \mathrm{~nm}^{21}$ were achieved, which is close to the distance where the deep sub-wavelength regime occurs (typically 10-20 nm $)^{20}$. However, this configuration allows near-field heat transfer only over a small area at the tip of the sphere, and is hence not practical for energy generation applications where the power is proportional to the area.

Here we show radiative heat transfer in the deep sub-wavelength regime between two parallel structures using precise positioning with integrated microelectromechanical actuators (MEMS). Our system relies on parallel nanobeams monolithically integrated with electrostatic comb drive actuators (Fig. 1). These actuators allow precise displacement control, limited in theory to sub- $\mathrm{nm}$ precision by Brownian motion and by actuation voltage uncertainty (see supplementary information S3). Their power consumption is also low $(<30 \mathrm{pW}$ in the ON state), more than three orders of magnitude lower than the typical heating power applied to the system.

We rely on the surface phonon-polariton resonance of $\mathrm{SiC}$ to create the surface waves responsible for near field heat transfer. Note that although there has been a lot of theory work on near-field heat transfer with thin SiC films $s^{2,6,22-24}$, no experimental work has been reported. We use silicon carbide deposited by plasma enhanced chemical vapor deposition (PECVD) and annealed to create a microcrystalline ( $\mu$-SiC) phase (see methods). We characterize the infrared permittivity $(\varepsilon)$ of $\mu$ $\mathrm{SiC}$ for the first time and find it to be well described by a Lorentz-Drude relation (equation 1) that has a sharper infrared resonance than most commonly used $\mathrm{SiO}_{2}$ :

$$
E(w)=E_{00}\left(1+\frac{w_{\mathrm{L}}-\mathrm{w}_{\mathrm{T}}}{\mathrm{w}_{\mathrm{T}}^{2}-\mathrm{w}^{2} \text {-irw }}\right),
$$

with $\omega_{T}=789 \mathrm{~cm}-1, \omega_{L}=956 \mathrm{~cm}-1, \varepsilon_{\infty}=8, \Gamma=20 \mathrm{~cm}-1$ (see characterization and extended discussion in supplementary information $\mathrm{S} 1$.)

We simulate the heat transfer between the nanobeams using a Fourier modal method based on the fluctuational electrodynamics formalism ${ }^{25,26}$ and we predict that the deep sub-wavelength regime occurs at distances $<200 \mathrm{~nm}$. The exact geometry of the nanobeams considered for this simulation is presented in supplementary information S2. For distances smaller than $200 \mathrm{~nm}$ (see Fig $1 \mathrm{~b}$ ) the simulated results follow the typical $1 / d^{\alpha}$ law that is characteristic of the deep sub- wavelength regime. The geometry dependent factor $\alpha=1.75$ in this case is intermediate between the parallel plate case $(\alpha=2)$ and the sphere-plane case $(\alpha=1.5)$. The simulations also show that heat transfer in the deep sub-wavelength regime is quasi-monochromatic (see Fig $1 \mathrm{c}$ ) and centered around the $\varepsilon(\omega)=-1$ surface phonon resonance frequency of $\operatorname{SiC}\left(937 \mathrm{~cm}^{-1}\right)$. 

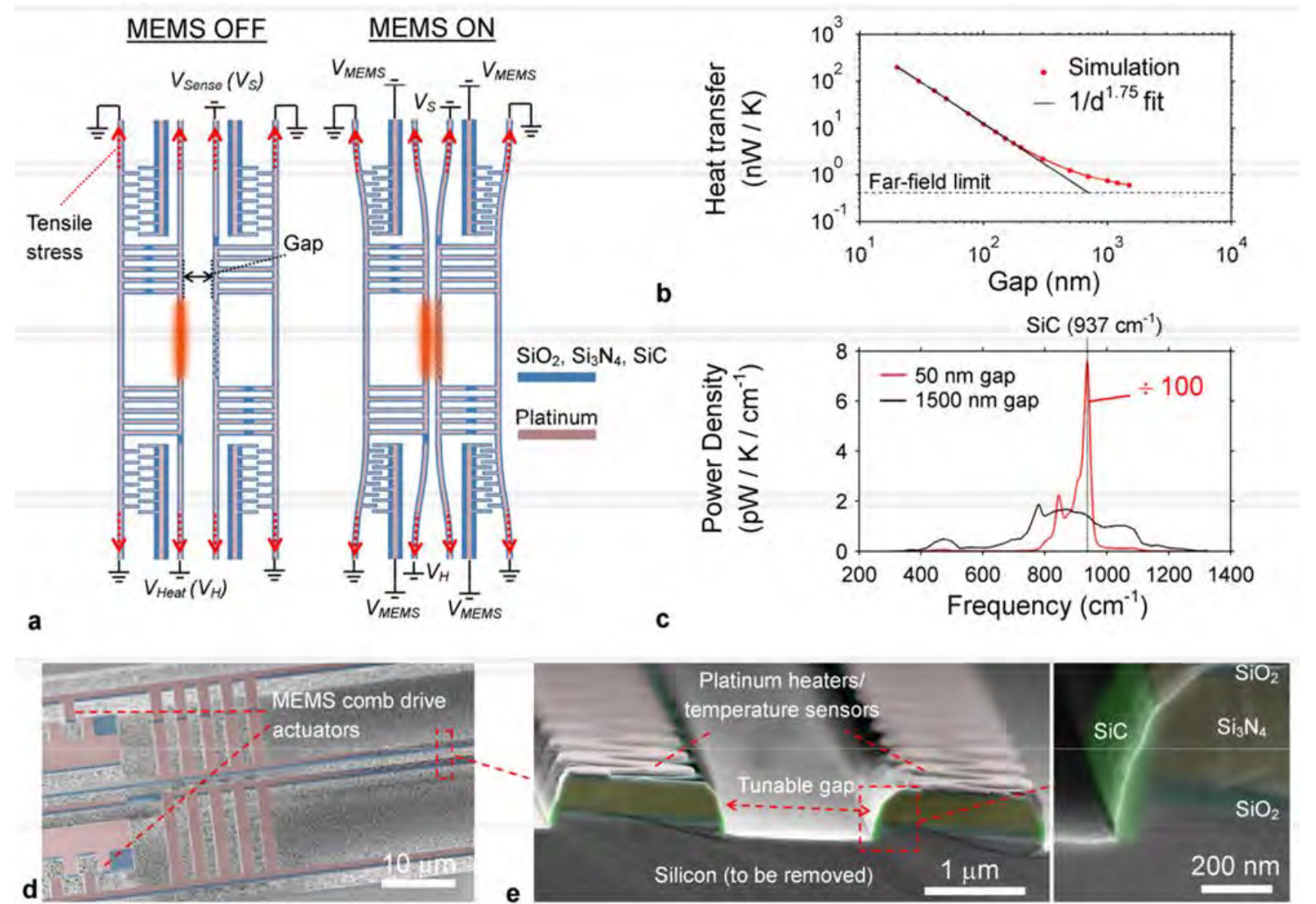

Figure 1 (a) Schematic of the device geometry (not to scale) and operation principle. (b) Simulated radiative heat transfer between two nanobeams as a function of the distance. The hot beam is set at $T=10 \mathrm{~K}$ above room temperature. The deep sub-wavelength regime occurs for beam separation below $200 \mathrm{~nm}$, where the heat transfer begin to scale as $1 / d 1.75$ (c) Simulated heat transfer spectrum between the nanobeams at two different separation. For $d=50$ $\mathrm{nm}$, the heat transfer is concentrated mainly around the SiC surface resonance (near $\omega=937 \mathrm{~cm}-1$ ). The secondary peak (at $\omega=844 \mathrm{~cm}-1$ ) results from a contribution of Si3N4 to the heat transfer. (d) False color scanning electron micrograph (SEM) of the device after structural release. (e) SEM false color cross section view of the nanobeams prior to structural release.

In order to achieve experimentally very small distances over large areas, it is critical that the two surfaces are completely parallel. Therefore no buckling should occur under changes of temperature. We prevent buckling by designing the beams such that they preserve the high tensile stress of silicon nitride ( $\sim 900 \mathrm{MPa}$ ) after structural release (see Fig. 1 a). This stress prevents thermal bimorph effect from causing buckling that would catastrophically impact the minimum achievable distance in our system at high temperatures (see Fig. 2). Our design also suppresses stress induced deformation that prevented us from reaching the deep sub-wavelength regime with our previous platform ${ }^{27}$. 


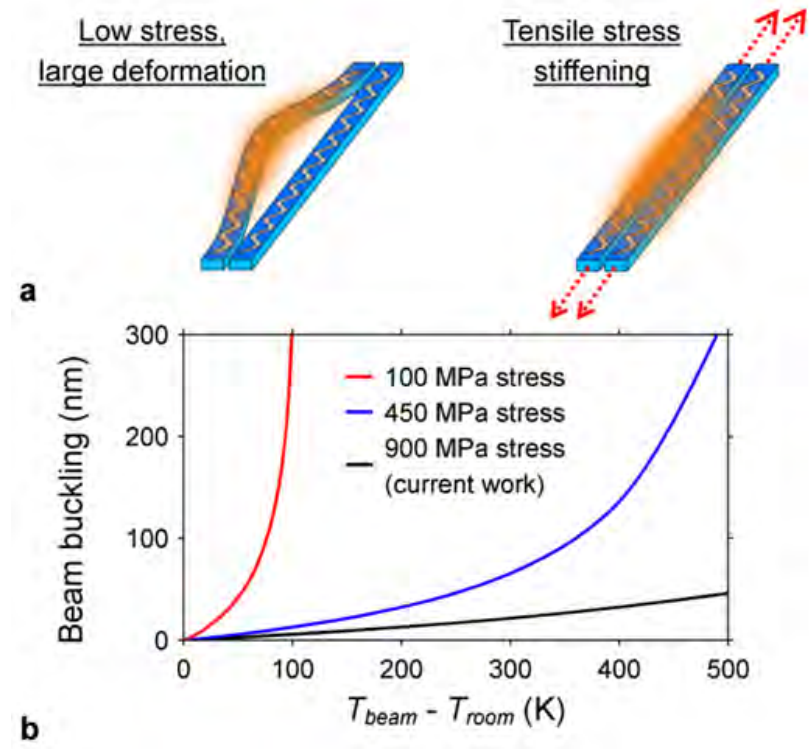

Figure 2 (a) Schematic of nanobeam buckling due to thermal bimorph effect. (b) Simulated buckling of a doubly clamped, $600 \mu \mathrm{m}$ long, bi-material beam made of Si3N4 (300 nm thick) and $\mathrm{Pt}(50 \mathrm{~nm}$ thick).

In order to measure the heat transfer, the beams are brought together by sweeping the voltage supplied to the MEMS actuator (see VMEMS in Fig. 1 a) while the temperature of the heated beam (Theat) and the sensing beam (Tsens) are measured (see Methods). The result of this scan is presented in Fig. 3 (b), where the inset shows the transition between near-field and contact regimes. The displacement of the nanobeams as a function of the MEMS voltage is also measured in a separate experiment (supplementary information S3) and presented in Fig 3 (a).

The experimental data of Fig 3 is converted to normalized heat transfer power (q, in W/K) using

$$
q=\frac{X P_{\text {heat }} T_{\text {sens }}}{\left(T_{\text {heat }}-T_{\text {sens }}\right)\left(T_{\text {heat }}+X T_{\text {sens }}\right)}
$$

where $\left(X=\sigma_{\text {sens }} / \sigma_{\text {heat }}\right)$ is the ratio of the background heat conduction $(\sigma)$ of the two nanobeams, and $P_{\text {heat }}$ is the supplied heating power. From the symmetry of the system, $X \approx 1$ for scans of relatively low temperature amplitude such as in Fig 3 (b). 


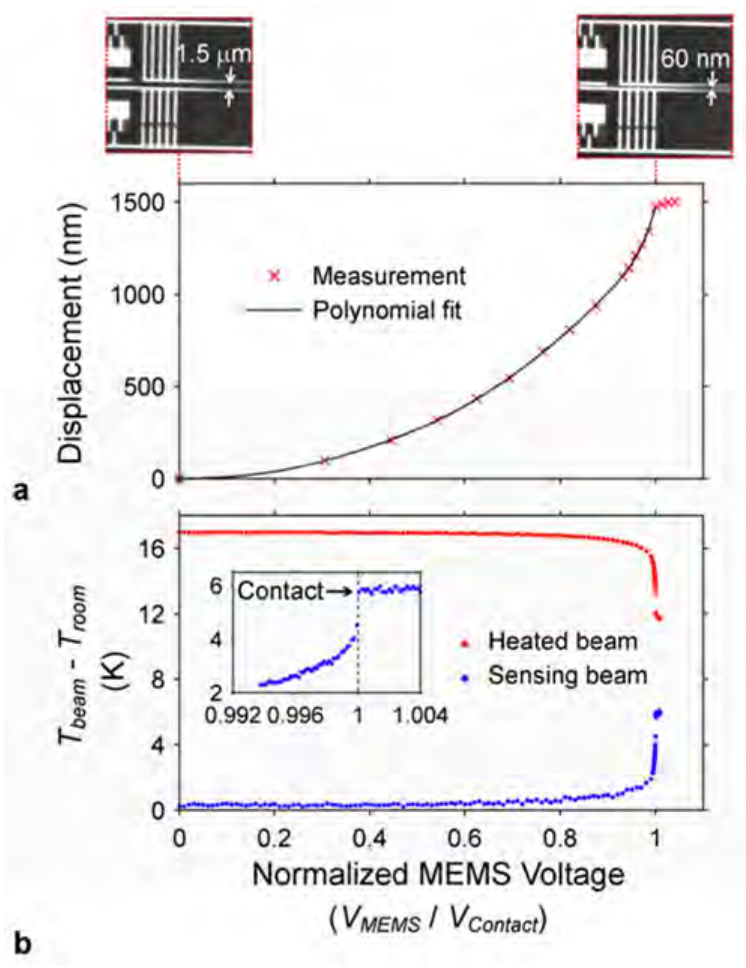

Figure 3 (a) Measured and fitted displacement of the nanobeams as a function of the voltage on the MEMS actuator. (b) Temperature of the heated beam (Theat) and the sensing beam $\left(T_{\text {Sens }}\right)$ as a function of the voltage on the MEMS actuator.

The measured heat transfer agrees well with the simulated values. In Fig 4 (a), the experimental heat transfer power is plotted and compared with the simulated data (which is fitted vertically and horizontally, see methods). At gaps larger than $\sim 150 \mathrm{~nm}$, the experimental values are slightly larger than the simulated ones. This could be caused by larger infrared material absorption coefficient (of, e.g., $\mathrm{Si}_{3} \mathrm{~N}_{4}$ ) than considered in the simulations, which would increase the contribution of propagating wave to the heat transfer. This increase causes the deep sub- wavelength regime to occur at $<150 \mathrm{~nm}$ distances, rather than $<200 \mathrm{~nm}$ in the simulated data (Fig $1 \mathrm{~b}$ ). At gaps smaller than $150 \mathrm{~nm}$, the experimental data matches the simulation more closely, and small deviations between simulation and experiments are visible only in the logarithmic scale inset in Fig. 4 (a). These are likely caused by the inability of the polynomial fit to perfectly match the experimental MEMS displacement in Fig 3 (a), or by slightly different experimental conditions between the MEMS displacement measurement (supplementary information S3) and the heat transfer measurement. 

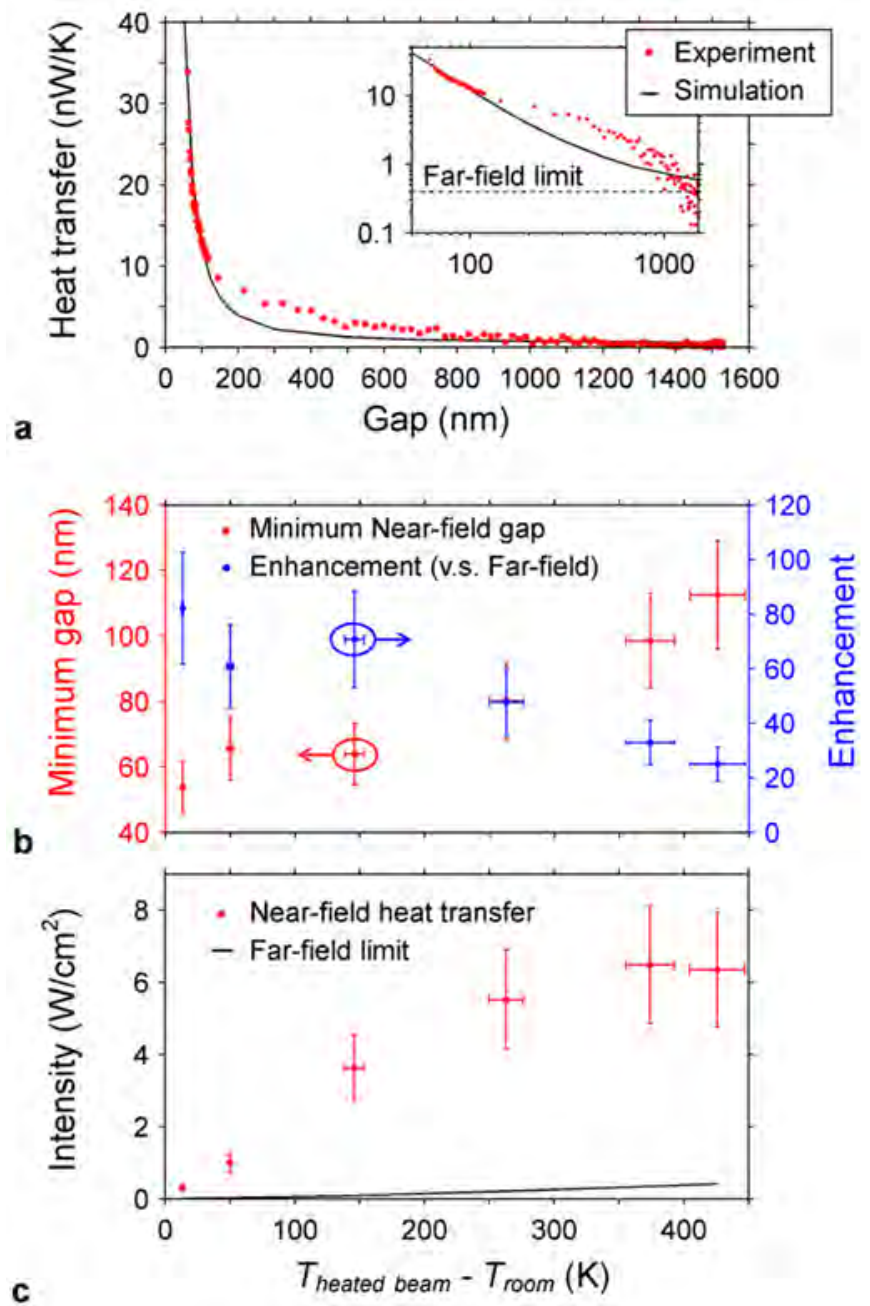

Figure 4 (a) Measured and simulated heat transfer between the nanobeams as a function of separation. The inset shows the same result, in logarithmic scale. (b) Minimum gap (i.e., before the beam come into contact) and maximum enhancement (relative to the far-field limit) achieved as a function of the heated beam temperature. This figure shows our ability to maintain the beam separation in the deep sub-wavelength regime (gap $<150 \mathrm{~nm}$ ) for beam temperatures as high as $720 \mathrm{~K}$. The error bars are calculated from the uncertainty on the thermal coefficient of resistance and the effective beam interaction length (see supplementary information S2). (c) Near-field heat transfer intensity (power per unit area) as a function of the temperature of the heated beam. The area is taken as the inner $\mathrm{SiC}$ surface of the nanobeams.

We achieve an $82 \times$ enhancement of heat transfer over the far-field limit and are able to maintain the nanobeams in the deep sub-wavelength regime for temperature gradients as high as $260 \mathrm{~K}$. The highest heat transfer value achieved in Fig 4 (a) is $34 \mathrm{nW} / \mathrm{K}$, which is $82 \times$ larger than the beam farfield radiation limit $(0.42 \mathrm{nW} / \mathrm{K})$. We define this limit as the maximum power that the $\mathrm{SiC}$ surface of the nanobeam can radiate to the far-field, at a given temperature (see Methods). This enhancement corresponds to a $54 \mathrm{~nm}$ gap between the nanobeams, which is well within the deep sub-wavelength regime $(<150 \mathrm{~nm})$. The measurement of Fig 4 (a) is repeated for different temperatures of the heated beam. In Fig 4 (b), the minimum achieved gap (i.e. the smallest nearfield gap achieved before the beams come into contact with each other) is reported as a function of the heated beam temperature. The minimum achieved near-field gap is found to increase slightly 
with temperature. This is most likely due to thermal buckling that occurs despite the large stiffness of the nanobeams. The increase is indeed consistent with the simulated buckling presented in Fig 2. We reach the deep sub-wavelength regime (i.e. distance $<150 \mathrm{~nm}$ ) with heated beam temperatures as high as $720 \mathrm{~K}$ (i.e. $425 \mathrm{~K}$ above room temperature). In this case the temperature gradient between the beams is $260 \mathrm{~K}$ (see supplementary information S4). Such high thermal gradient translates to a high ( $>6 \mathrm{~W} / \mathrm{cm}^{2}$, see Fig $4 \mathrm{c}$ ) net energy flux which, for applications such as energy generation, is often more crucial than the relative enhancement itself.

We achieved the first demonstration of near-field radiative heat transfer between parallel objects in the deep sub-wavelength regime and at high temperature gradient. This nanoscale approach offers a clear path towards applications of near-field heat transfer such as near-field thermophotovolatic energy generation. We also note that our approach could be scaled up to a larger effective area by simply arraying several nanobeams (atop, e.g., a photovoltaic cell) and by individually controlling their out-of-plane displacement using MEMS actuators.

\section{Methods}

Microcrystalline Silicon Carbide $(\mu$-SiC) deposition

Amorphous silicon carbide is first deposited in by plasma enhanced chemical vapor deposition (PECVD) from silane $\left(\mathrm{SiH}_{4}\right)$ and methane $\left(\mathrm{CH}_{4}\right)$ gas precursors in a 1:20 ratio and with argon as a dilution gas. The film is then annealed in argon atmosphere at $1100{ }^{\circ} \mathrm{C}$ for 90 minutes to grow a microcrystalline phase ${ }^{28}$. The silane/methane concentration ratio was optimized by measuring the refractive index of the film by ellipsometry after the anneal process. The 1:20 ratio yields a 2.53 refractive index (at $\lambda=1500 \mathrm{~nm}$ ), which is very close to the theoretical value (2.57). Higher or lower ratio both lead to higher refractive index, which is consistent with the growth of silicon or carbon clusters during annealing of non-stoichiometric films.

Device fabrication

A $\mathrm{SiO}_{2} / \mathrm{Si}_{3} \mathrm{~N}_{4} / \mathrm{SiO}_{2}$ film stack is first deposited on a conventional silicon wafer by low pressure chemical vapor deposition (LPCVD). The structure is then defined by deep-UV lithography and anisotropically etched in fluorine chemistry. $\mathrm{SiC}$ is then deposited over the structure by PECVD an annealed at $1100{ }^{\circ} \mathrm{C}$ to grow the $\mu$-SiC phase. SiC is anisotropically etched in fluorine chemistry, such that $\mu$-SiC remains only on the nanobeam sidewalls. Platinum heaters and metal contact are deposited be electron beam evaporation and lift-off using a chrome adhesion layer. The device is finally released by undercutting the silicon substrate in $\mathrm{XeF}_{2}$ chemistry.

\section{Experimental condition \& procedure}

The heat transfer experiments are performed at room temperature in a high vacuum $\left(9 \times 10^{-5}\right.$ torr $)$ electrical probe station. Electrical measurements are performed using an Agilent B1500 semiconductor parameter analyzer. The MEMS voltage $\left(V_{M E M S}\right)$ is swept to bring the two nanobeams together while constant heating $\left(V_{\text {Heat }}\right)$ and sensing $\left(V_{\text {Sens }}\right)$ voltages are supplied to each of the two nanobeams. The time interval between each MEMS voltage increment $(50 \mathrm{~ms})$ is several times larger than the thermal response time of the system $(7 \mathrm{~ms})$, such that all measurements are in steady state. $V_{\text {Sens }}$ is kept much lower than $V_{\text {Heat }}$, such that the power supplied to the sensing beam is always at least 25 times lower than the power supplied to the heated beam $\left(P_{\text {Heat }}\right)$. These constant 
voltages are also used to measure the temperature of the two beams, through the variation their electrical resistance $(R)$ as $\Delta R / R=T C R \times \Delta T$, where $T C R=0.00166 \mathrm{~K}^{-1}$ is the measured temperature coefficient of resistance of platinum (see next section).

\section{Temperature coefficient of resistance measurement}

The temperature coefficient of resistance $(T C R)$ of Platinum is measured by placing the device on a hot plate ramped from room temperature to $100^{\circ} \mathrm{C}$. We observe that the $T C R$ differs greatly after the device is used at high temperature for the first time. For this reason, prior to TCR measurement, a heating voltage $\left(V_{\text {Heat }}\right) 10 \%$ higher than the one used for the highest temperature point in Fig $4(\mathrm{~b}$, c) is supplied to the device under vacuum and held for 5 minutes until the current (and hence the electrical resistance) stabilizes. After this procedure, the device is placed on a hot plate ramped from room temperature to $100{ }^{\circ} \mathrm{C}$ and the resistance is measured at every $3{ }^{\circ} \mathrm{C}$ increment. We find that the thermal coefficient of resistance is $0.00125 \mathrm{~K}^{-1}$ before the annealing procedure and $T C R=$ $0.00166 \mathrm{~K}^{-1}$ after. We estimate a $\pm 5 \%$ error on this value from the repeatability of the measurement.

\section{Fitting procedure}

In Fig 3 (a) the measured displacement as a function of the MEMS voltage is fitted using an 8th order polynomial. The fitted function is subsequently used to convert the MEMS voltage to displacement values. The polynomial order is chosen iteratively to be high enough to match the experimental points well, while being low enough to minimize spurious oscillation between the experimental points.

In Fig 4 (a) the experimental and theoretical data are fitted together in two different ways. Firstly, the experimental data is translated horizontally to account for the uncertainty on the initial gap between the nanobeams (i.e., the relative displacement of the beam is well known from Fig 3 (a), but the exact initial separation is unknown). The initial gap that best fit the experimental results is $1529 \mathrm{~nm}$, close to the designed $1500 \mathrm{~nm}$ value. Secondly, the experimental data is translated vertically to account for parasitic heat conduction through the substrate. The translation that best fits the experiment is $1 \mathrm{nW} / \mathrm{K}$, which is negligible compared to the achieved radiative heat achieved at the smallest gap $(34 \mathrm{nW} / \mathrm{K})$.

Far-field limit calculation

We define the far-field limit as the maximum power that can be radiated to the far-field by the inner Silicon Carbide part of the heated nanobeam. This calculation is performed using the Fourier modal method ${ }^{25,26}$. The far-field temperature in this simulation is set to room temperature $(293 \mathrm{~K})$. 


\section{List of supplemental material}

S1 - Microcrystalline silicon carbide ( $\square \mathrm{SiC}$ ) infrared characterization S2 - System dimensions S3 - MEMS displacement measurement and theoretical precision limit S4 - High temperature experimental data

\section{Acknowledgements}

The authors gratefully acknowledge support from DARPA for award FA8650-14-1-7406 supervised by Dr. Avram Bar-Cohen. This work made use of the Cornell Center for Materials Research Shared Facilities, which are supported through the NSF MRSEC program (DMR1120296), and of the Cornell NanoScale Facility, a member of the National Nanotechnology Infrastructure Network, which is supported by the National Science Foundation (Grant ECCS0335765). R.S.-G. held subsequent postdoctoral fellowships from the Fonds de recherche du Québec-Nature et Technologies, and from the Natural Sciences and Engineering Research Council of Canada (NSERC) during this work.

\section{Competing financial interest}

The authors declare no competing financial interests.

\section{References}

1 DiMatteo, R. S. et al. Enhanced photogeneration of carriers in a semiconductor via coupling across a nonisothermal nanoscale vacuum gap. Applied Physics Letters 79, 1894-1896, (2001).

2 Narayanaswamy, A. \& Chen, G. Surface modes for near field thermophotovoltaics. Applied Physics Letters 82, 3544-3546, (2003).

3 Laroche, M., Carminati, R. \& Greffet, J.-J. Near-field thermophotovoltaic energy conversion. J Appl Phys 100, 063704, (2006).

4 Park, K., Basu, S., King, W. P. \& Zhang, Z. M. Performance analysis of near-field thermophotovoltaic devices considering absorption distribution. Journal of Quantitative Spectroscopy and Radiative Transfer 109, 305-316, (2008).

$5 \quad$ Ilic, O., Jablan, M., Joannopoulos, J. D., Celanovic, I. \& Solja?i, M. Overcoming the black body limit in plasmonic and graphene near-field thermophotovoltaic systems. Optics Express 20, A366-A384, (2012).

6 Otey, C. R., Lau, W. T. \& Fan, S. Thermal rectification through vacuum. Phys Rev Lett 104, 154301 (2010).

7 Wang, L. P. \& Zhang, Z. M. Thermal rectification enabled by near-rield radiative heat transfer between intrinsic silicon and a dissimilar material. Nanosc Microsc Therm 17, 337-348, (2013).

8 Ben-Abdallah, P. \& Biehs, S.-A. Near-field thermal transistor. Phys Rev Lett 112, 044301 (2014).

9 Hu, L., Narayanaswamy, A., Chen, X. \& Chen, G. Near-field thermal radiation between two closely spaced glass plates exceeding Planck’s blackbody radiation law. Applied Physics Letters 92, 133106, (2008).

10 Ijiro, T. \& Yamada, N. Near-field radiative heat transfer between two parallel SiO2 plates with and without microcavities. Applied Physics Letters 106, (2015).

11 Ito, K., Miura, A., Iizuka, H. \& Toshiyoshi, H. Parallel-plate submicron gap formed by micromachined low-density pillars for near-field radiative heat transfer. Applied Physics Letters 106, 083504, (2015).

12 Kralik, T. et al. Strong near-Field enhancement of radiative heat transfer between metallic surfaces. Phys Rev Lett 109, 224302 (2012).

13 Ottens, R. S. et al. Near-field radiative heat transfer between macroscopic planar surfaces. Phys Rev Lett 107, 014301 (2011).

14 Cravalho, E. G., Tien, C. L. \& Caren, R. P. Effect of small spacings on radiative transfer between two dielectrics. Journal of Heat Transfer 89, 351-358, (1967).

15 Polder, D. \& Van Hove, M. Theory of radiative heat transfer between closely spaced bodies. Phys Rev B 4, 3303-3314 (1971).

16 Shchegrov, A. V., Joulain, K., Carminati, R. \& Greffet, J.-J. Near-field spectral effects due to electromagnetic surface excitations. Physical Review Letters 85, 1548-1551 (2000).

17 Mulet, J.-P., Joulain, K., Carminati, R. \& Greffet, J.-J. Enhance radiative heat transfer at nanometric 
distances Microscale Thermophysical Engineering 6, 209-222, (2002).

18 Iizuka, H. \& Fan, S. Rectification of evanescent heat transfer between dielectric-coated and uncoated silicon carbide plates. J Appl Phys 112, 024304, (2012).

19 Shen, S., Narayanaswamy, A. \& Chen, G. Surface phonon polaritons mediated energy transfer between nanoscale gaps. Nano Lett 9, 2909-2913, (2009).

20 Rousseau, E. et al. Radiative heat transfer at the nanoscale. Nat Photon 3, 514-517, (2009).

21 Song, B. et al. Enhancement of near-field radiative heat transfer using polar dielectric thin films. Nat Nano 10, 253-258, (2015).

22 Francoeur, M., Mengüç, M. P. \& Vaillon, R. Near-field radiative heat transfer enhancement via surface phonon polaritons coupling in thin films. Appl Phys Lett 93, 043109, (2008).

23 Miller, O. D., Johnson, S. G. \& Rodriguez, A. W. Effectiveness of thin films in lieu of hyperbolic metamaterials in the near field. Physical Review Letters 112, 157402 (2014).

24 Basu, S., Yang, Y. \& Wang, L. Near-field radiative heat transfer between metamaterials coated with silicon carbide thin films. Applied Physics Letters 106, 033106, (2015).

25 Lussange, J. et al. Radiative heat transfer between two dielectric nanogratings in the scattering approach. Phys Rev B 86, 085432 (2012).

26 Otey, C. R., Zhu, L., Sandhu, S. \& Fan, S. Fluctuational electrodynamics calculations of near-field heat transfer in non-planar geometries: A brief overview. J Quant Spectrosc RA 132, 3-11, (2014).

27 St-Gelais, R., Guha, B., Zhu, L., Fan, S. \& Lipson, M. Demonstration of strong near-field radiative heat transfer between integrated nanostructures. Nano Letters (2014).

28 Calcagno, L., Musumeci, P., Roccaforte, F., Bongiorno, C. \& Foti, G. Crystallization process of amorphous silicon-carbon alloys. Thin Solid Films 411, 298-302, (2002). 


\title{
Supplementary Information: Near-field radiative heat transfer between nanostructures in the deep sub-wavelength regime
}

\author{
Raphael St-Gelais, ${ }^{1,2}$ Linxiao Zhu, ${ }^{3}$ Shanhui Fan, ${ }^{3}$ and Michal Lipson ${ }^{*}, 1,2$ \\ ${ }^{I}$ School of Electrical and Computer Engineering, Cornell University, Ithaca, New York 14853, United States \\ ${ }^{2}$ Department of Electrical Engineering, Columbia University, New York, New York 10027, United States \\ ${ }^{3}$ Ginzton Laboratory, Stanford University, Stanford, California 94305, United States \\ *e-mail:ml3745@columbia.edu
}

\section{S1 - Microcrystalline silicon carbide $(\mu-\mathrm{SiC})$ infrared characterization}

We characterize the infrared permittivity of $\mu$-SiC by measuring the absorption of a $120 \mathrm{~nm}$ thick film deposited on a conventional silicon substrate. The film is deposited and annealed following the procedure described in Methods. We measure the absorption at 20 degrees grazing incidence using a Fourier transform infrared spectrometer (Bruker Hyperion). At grazing (rather than normal) incidence, measurements are sensitive to absorption from longitudinal optical phonons (LO), as well as from transverse optical (TO) phonons (which are usually the only ones visible in normal incidence spectra.) From this measurement (see Fig S1 a) we are able to fit the absorption of the film using conventional one-dimensional optical multilayer calculations and a Lorentz-Drude model for the complex permittivity of $\mu$-SiC,

$$
\varepsilon(\omega)=\varepsilon_{\infty}\left(1+\frac{\omega_{L}^{2}-\omega_{T}^{2}}{\omega_{T}^{2}-\omega^{2}-i \Gamma \omega}\right),
$$

where $\omega_{L}$ and $\omega_{T}$ are the LO and TO phonon frequencies, $\varepsilon_{\infty}$ is the baseline permittivity, and $\Gamma$ is the phonon damping factor. For the simulation, we set the refractive index of the substrate to $n_{\mathrm{Si}}=3.42$, since silicon is transparent in this frequency range. The numbers that best fit the simulations are $\omega_{T}=789 \mathrm{~cm}^{-1}, \omega_{L}=956 \mathrm{~cm}^{-1}, \varepsilon_{\infty}=8$, and a damping factor $\left(\Gamma=20 \mathrm{~cm}^{-1}\right)$ that is 5 times larger than what is reported for single crystal silicon carbide [S1]. Although the damping parameter is larger than for single crystal $\mathrm{SiC}, \mu$-SiC can still be expected to yield narrower nearfield heat transfer spectra than $\mathrm{SiO}_{2}$, which is currently the most widely used material for near- field heat transfer experiments. In Fig S1 (b), we compare the local density of states (LDOS),

$$
L D O S \propto \frac{\varepsilon^{\prime \prime}}{|1+\varepsilon|^{2}}
$$

at surfaces of $\mu$-SiC and $\mathrm{SiO}_{2}$, and find that $\mu$-SiC can be expected to yield a $5.5 \times$ narrower heat transfer frequency distribution than $\mathrm{SiO}_{2}$. For this simulation, permittivity of $\mathrm{SiO}_{2}$ is taken from $[\mathrm{S} 2]$. 

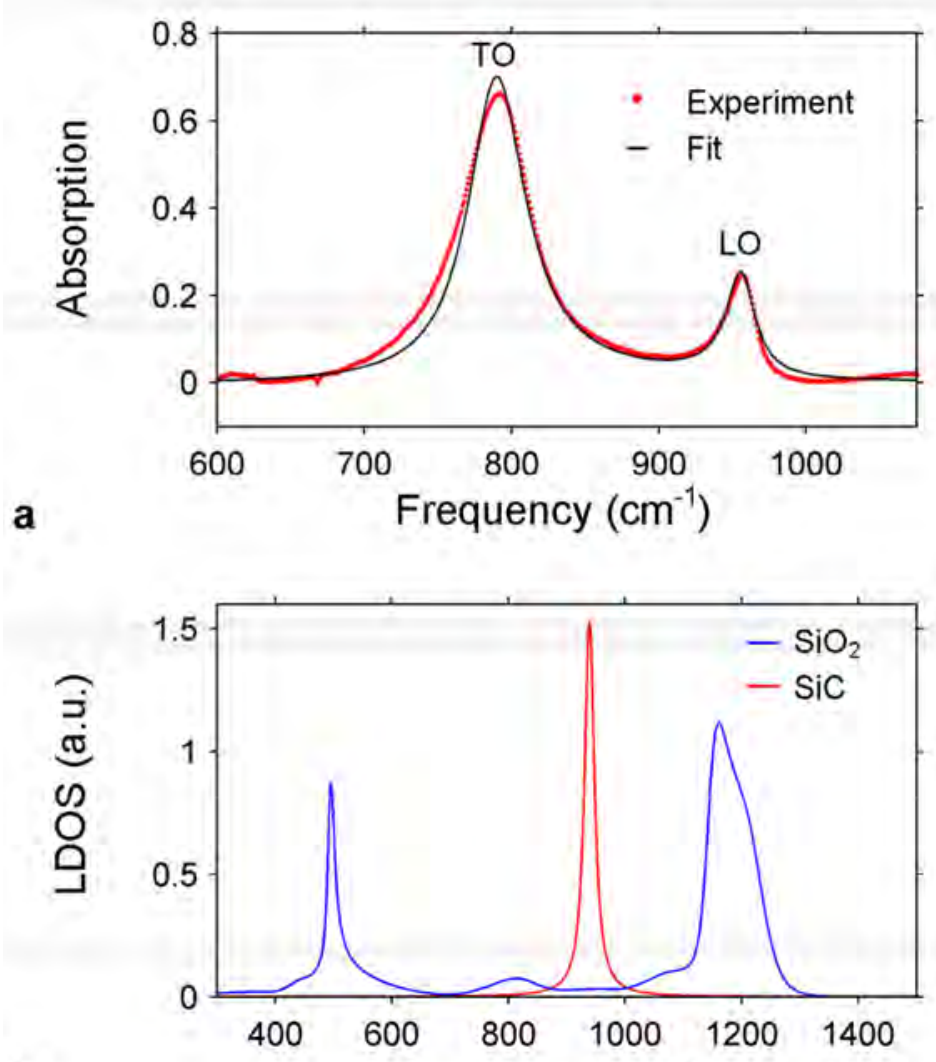

b

Frequency $\left(\mathrm{cm}^{-1}\right)$

Figure S1. (a) Measured and fitted infrared absorption spectra of a $120 \mathrm{~nm}$ thin $\mu$-SiC film on silicon at 20 degree grazing incidence. (b) Comparison of the local density of states at the surfaces of $\mu-\mathrm{SiC}$ and $\mathrm{SiO}_{2}$.

\section{S2 - System dimensions}

The cross section dimensions of the nanobeam considered for the near-field simulations are presented in Fig. S2. The dimensions of the MEMS are presented in Fig. S3. From the dimensions in Fig. S3, we estimate a 25\% uncertainty on the effective beam interaction length due to the length difference between the beams parallel regions $(200 \mu \mathrm{m})$ and the heater length $(155 \mu \mathrm{m})$.

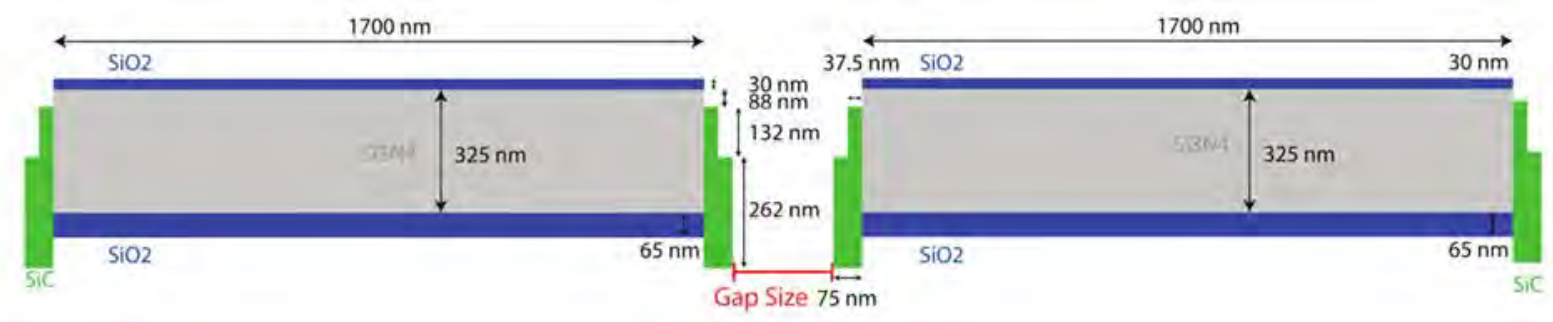

Figure S2. Beam cross-section considered for the simulations.

Approved for public release; distribution unlimited. 


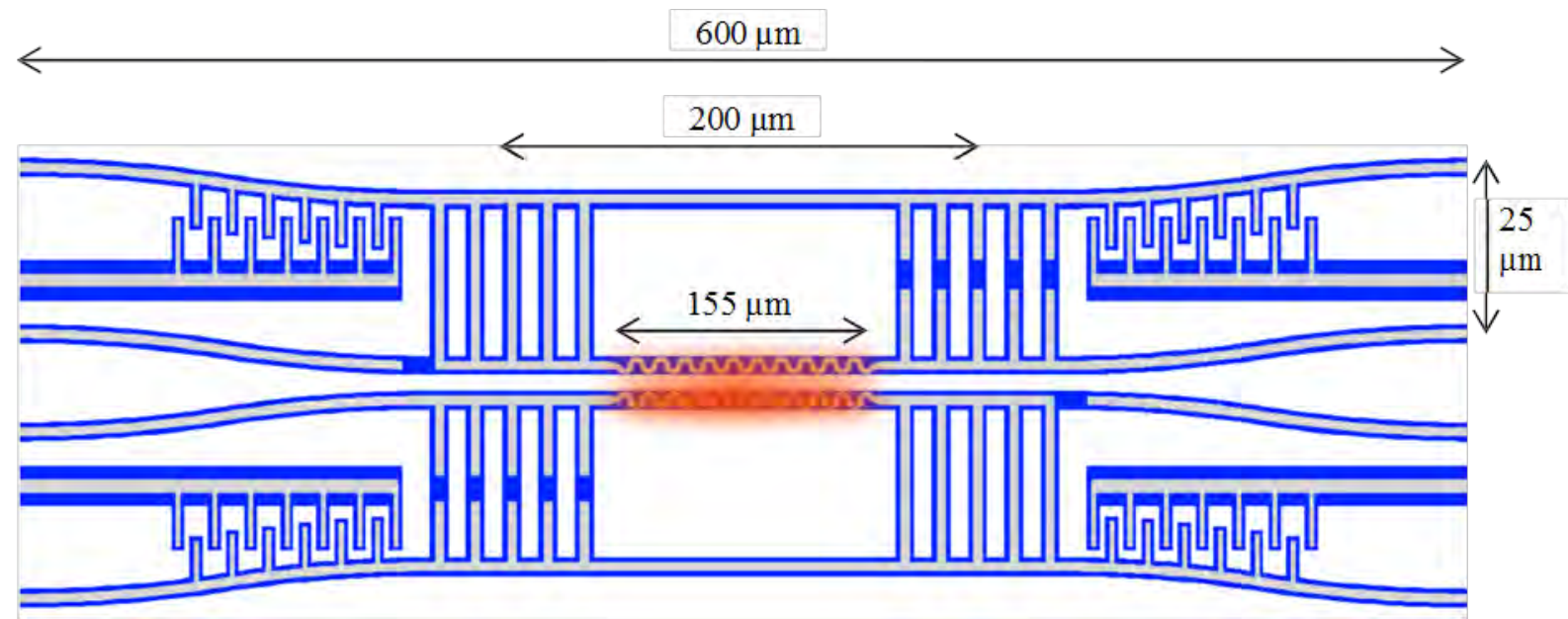

Figure S3. Main dimensions of the MEMS structure (drawing not to scale).

\section{S3 - MEMS displacement measurement and theoretical precision limit}

The MEMS displacement as a function of the applied voltage is measured by placing the device on an electrical probe station equipped with a high magnification (50X) microscope. The displacement is measured as a function of the voltage applied on the MEMS actuator using an image treatment algorithm that fits Gaussian functions over each optical image of the nanobeams. The displacement is then evaluated by calculating the centroid of the fitted Gaussian functions.

The highest measured displacement sensitivity of the MEMS (see main paper, Fig 3 a) is 65 $\mathrm{nm} / \mathrm{V}$ when the nanobeams are almost in contact (i.e., close to $V_{\text {contact }}=155 \mathrm{~V}$ ). The resolution of Agilent B1500 voltage source being $10 \mathrm{mV}$, this translates to a $0.65 \mathrm{~nm}$ resolution limit on the MEMS displacement. Fundamentally, the resolution on the MEMS displacement would eventually be limited by thermal mechanical motion $\left(x_{r m s}\right)$ given by [S3]:

$$
x_{R M S}=\sqrt{\frac{k_{B} T}{K}},
$$

where $K$ is the mechanical stiffness of the structures, which we estimate to $1-2 \mathrm{~N} / \mathrm{m}$. In this case, and considering the highest temperature used in our experiments, the amplitude of thermal mechanical vibration would be around $0.1 \mathrm{~nm}$ RMS.

\section{S4 - High temperature experimental data}

Figure S4 presents the same temperature experimental data as in the main manuscript (Fig. $3 \mathrm{~b}$ ), but for the highest temperature used in this work. At the smallest gap between the nanobeams (before contact), the thermal gradient is $261 \mathrm{~K}$. 
We note that in this case the two curves are asymmetric. This is caused by a higher background conduction $(\sigma)$ of the heated beam compared to the background conduction of the sensing beam (i.e. $X=\sigma_{\text {sens }} / \sigma_{\text {heat }} \neq 1$, unlike for the low temperature scan in Fig $3 \mathrm{~b}$ ). In the present case the ratio is $X=0.28$. This is most likely caused by a higher far-field radiation of the heated MEMS compared to the sensing one when the temperature difference is very high.

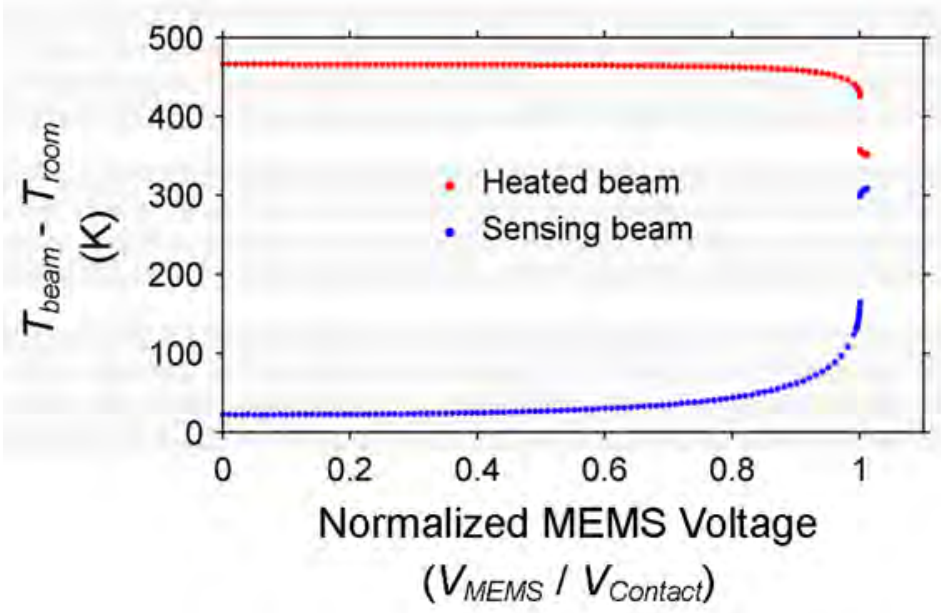

Figure S4. Measured temperature of the heated and sensing beam as a function of the MEMS voltage for the highest temperature scan performed in this work.

\section{References}

[S1] Palik, E. D. Handbook of optical constants of solids. Vol. 3 (Academic press, 1998)

[S2] Kitamura, R., Pilon, L. \& Jonasz, M. Optical constants of silica glass from extreme ultraviolet to far infrared at near room temperature. Appl. Opt. 46, 8118-8133, doi:10.1364/ao.46.008118(2007).

[S3] Gabrielson, T. B. Mechanical-thermal noise in micromachined acoustic and vibration sensors. Electron Devices, IEEE Transactions on 40, 903-909, doi:10.1109/16.210197 (1993). 


\section{List of Acronyms, Abbreviations, and Symbols}

$\begin{array}{ll}\text { ACRONYM } & \text { DESCRIPTION } \\ \mathrm{CH}_{4} & \text { methane } \\ \text { LPCVD } & \text { low pressure chemical vapor deposition } \\ \text { MEMS } & \text { micro-electromechanical system } \\ \text { PECVD } & \text { plasma enhanced chemical vapor deposition } \\ \mathrm{Pt} & \text { platinum } \\ \mathrm{SEM} & \text { scanning electron micrograph } \\ \mathrm{Si}_{3} \mathrm{~N}_{4} & \text { silicon nitride } \\ \mathrm{SiC} & \text { silicon carbide } \\ \mathrm{SiH}_{4} & \text { silane } \\ \mathrm{SiO}_{2} & \text { silicon dioxide } \\ \mathrm{T}_{\text {fix }} & \text { fixed heated beam } \\ \mathrm{T}_{\text {mob }} & \text { mobile sensing beam }\end{array}$

